\title{
Big projective modules over noetherian semilocal rings
}

\author{
Dolors Herbera* \\ Departament de Matemàtiques, \\ Universitat Autònoma de Barcelona, \\ 08193 Bellaterra (Barcelona), Spain \\ e-mail: dolors@mat.uab.cat \\ Pavel Př́ihoda ${ }^{\dagger}$ \\ Charles University, Faculty of Mathematics and Physic, \\ Department of Algebra, Sokolovská 83, \\ 18675 Praha 8, Czech Republic \\ e-mail: prihoda@karlin.mff.cuni.cz
}

\begin{abstract}
We prove that for a noetherian semilocal ring $R$ with exactly $k$ isomorphism classes of simple right modules the monoid $V^{*}(R)$ of isomorphism classes of countably generated projective right (left) modules, viewed as a submonoid of $V^{*}(R / J(R))$, is isomorphic to the monoid of solutions in $\left(\mathbb{N}_{0} \cup\{\infty\}\right)^{k}$ of a system consisting of congruences and diophantine linear equations. The converse also holds, that is, if $M$ is a submonoid of $\left(\mathbb{N}_{0} \cup\{\infty\}\right)^{k}$ containing an order unit $\left(n_{1}, \ldots, n_{k}\right)$ of $\mathbb{N}_{0}^{k}$ which is the set of solutions of a system of congruences and linear diophantine equations then it can be realized as $V^{*}(R)$ for a noetherian semilocal ring such that $R / J(R) \cong M_{n_{1}}\left(D_{1}\right) \times \cdots \times M_{n_{k}}\left(D_{k}\right)$ for suitable division rings $D_{1}, \ldots, D_{k}$.
\end{abstract}

A theorem of Kaplansky states that, for any ring $R$, a projective right $R$-module is a direct sum of countably generated projective right $R$-modules. This reduces the study of direct summands of $R^{(I)}$, where $I$ denotes an arbitrary set, to the study of direct sum decomposition of $R^{(\omega)}$ or, equivalently, to the study of countably generated projective right $R$-modules.

\footnotetext{
*Partially supported by MEC-DGESIC (Spain) through Project MTM2005-00934, and by the Comissionat Per Universitats i Recerca de la Generalitat de Catalunya through Project 2005SGR00206.

This paper was started when both authors were working within the Research Programme on Discrete and Continuous methods of Ring Theory at the CRM, Barcelona (Spain). They thank their host for its hospitality.

$\dagger$ At CRM supported by grant SB 2005-0182 from MEC, recently supported by research project MSM 0021620839.
} 
The commutative monoid $V(R)$ of isomorphism classes of finitely generated projective right $R$-modules, with the addition induced by the direct sum of modules, encodes the direct sum behavior of finite direct sums of finitely generated projective right $R$-modules. Similarly, the monoid $V^{*}(R)$ of isomorphism classes of countably generated projective right $R$-modules, with the addition induced by the direct sum of modules, encodes the directsum behavior of countably generated projective modules. In this paper we characterize the monoids that can be realized as $V^{*}(R)$ for $R$ a noetherian semilocal ring.

A ring $R$ is said to be semilocal if it is semisimple artinian modulo its Jacobson radical $J(R)$. To fix notation, we assume that $R / J(R) \cong M_{n_{1}}\left(D_{1}\right) \times \cdots \times M_{n_{k}}\left(D_{k}\right)$ where $D_{1}, \ldots, D_{k}$ are division rings uniquely determined up to isomorphism. The monoids $V(R)$ and $V^{*}(R)$ can be viewed as submonoids of $V(R / J(R)) \cong \mathbb{N}_{0}^{k}$ and $V^{*}(R / J(R)) \cong\left(\mathbb{N}_{0} \cup\right.$ $\{\infty\})^{k}=\left(\mathbb{N}_{0}^{*}\right)^{k}$, respectively; the class of $R$ corresponds to the element $\left(n_{1}, \ldots, n_{k}\right) \in \mathbb{N}_{0}^{k}$. The submonoids of $\mathbb{N}_{0}^{k}$ containing $\left(n_{1}, \ldots, n_{k}\right)$ that can be realized as $V(R)$ for a semilocal ring $R$ were characterized in [10] as the set of solutions in $\mathbb{N}_{0}^{k}$ of systems of diophantine equations of the form

$$
D\left(\begin{array}{c}
t_{1} \\
\vdots \\
t_{k}
\end{array}\right) \in\left(\begin{array}{c}
m_{1} \mathbb{N}_{0}^{*} \\
\vdots \\
m_{n} \mathbb{N}_{0}^{*}
\end{array}\right) \quad \text { and } \quad E_{1}\left(\begin{array}{c}
t_{1} \\
\vdots \\
t_{k}
\end{array}\right)=E_{2}\left(\begin{array}{c}
t_{1} \\
\vdots \\
t_{k}
\end{array}\right)
$$

where the coefficients of the matrices $D, E_{1}$ and $E_{2}$ as well as $m_{1}, \ldots, m_{n}$ are elements of $\mathbb{N}_{0}$. Such submonoids of $\mathbb{N}_{0}^{k}$ are called full affine submonoids (cf. Definition 1.5 and Proposition 6.2). This terminology was introduced by Hochster in [19]. However full affine monoids appear in different contexts with different names. In the setting of commutative noetherian rings they are also called positive normal monoids, see [2]. Such monoids also appear in generalizations of the multiplicative ideal theory where they are called finitely generated Krull monoids see, for example, [6].

In this paper we show that the submonoids of $\left(\mathbb{N}_{0}^{*}\right)^{k}$ that can be realized as $V^{*}(R)$ for a noetherian semilocal ring $R$ are precisely the sets of solutions in $\left(\mathbb{N}_{0}^{*}\right)^{k}$ of systems of type (1). We refer the reader to Theorem 2.7 for the precise statement. Most of the paper is devoted to the proof of Theorem 2.7 which has, essentially, two quite different parts. A more ring theoretical one, in which we provide the necessary tools to construct noetherian semilocal rings with prescribed monoid $V^{*}(R)$. Our key idea is to use a well known theorem due to Milnor stating that, under mild restrictions, the category of right projective modules over a pullback of rings is equivalent to the pullback of the categories of projective modules. Surprisingly enough, just considering pullbacks of semilocal principal ideal domains (or just noetherian semilocal rings such that all projective modules are free) and semisimple artinian rings a rich supply of noetherian semilocal rings $R$ with non-trivial $V^{*}(R)$ is obtained.

The second part of the paper (and of the proof of Theorem 2.7) deals with submonoids of $\left(\mathbb{N}_{0}^{*}\right)^{k}$. Our starting point are the results in [23] where it was proven that, for a noetherian semilocal ring, $V^{*}(R)$ is built up from a finite collection of full affine submonoids of $\mathbb{N}_{0}^{r_{1}}, \ldots, \mathbb{N}_{0}^{r_{m}}$, respectively, where $r_{i} \leq k$, chosen in a compatible way. These monoids are placed in the finite supports of the elements of $V^{*}(R)$ or, better saying, in the complementary of the infinite supports of the elements of $V^{*}(R)$, see Definition 1.2 for the unexplained 
terminology. In the paper, we make an abstraction of this type of monoid by introducing the concept of full affine system of supports in Definition 7.1, then $V^{*}(R)$, viewed as a submonoid of $\left(\mathbb{N}_{0}^{*}\right)^{k}$, is given by a full affine system of supports. Our main result in this part of the paper shows that the monoids given by a full affine system of supports are precisely the solutions in $\left(\mathbb{N}_{0}^{*}\right)^{k}$ of systems of the form (1).

We stress the fact that though the description of these submonoids of $\left(\mathbb{N}_{0}^{*}\right)^{k}$ as sets of solutions of a system of equations is very elegant, and it extends nicely the characterization for the case of finitely generated projective modules, the one given by the systems of supports seems to give a better idea of the complexity of the monoids we are working with. The contrast with the commutative situation is quite striking, all projective modules over a commutative semilocal indecomposable ring are free [18. On the other hand, as we explain along the paper and especially in 98 (cf. Definition 1.7 and Examples 8.5), the noetherian situation is simpler than the general one.

Our interest on semilocal rings stems from the fact that many classes of small modules have a semilocal endomorphism ring. For example, artinian modules or, more generally, modules with finite Goldie and dual Goldie dimension [17, finitely presented modules over a local ring or, more generally, finitely presented modules over a semilocal ring are classes of modules with a semilocal endomorphism ring [14. We refer to the monograph [9] as a source to read about, the good and the not so good, properties of modules with a semilocal endomorphism ring.

A description of the projective modules over the endomorphism ring is a first step towards understanding (part of) the category $\operatorname{Add}(M)$ of direct summands of any direct sum of copies of $M$. Wiegand in 28] proved that all monoids of solutions in $\mathbb{N}_{0}^{k}$ of systems of the form (2) can be realized as $V(R)$ when $R$ is the endomorphism ring of a finitely presented module over a noetherian semilocal ring, or the endomorphism ring of an artinian module. Yakovlev [30, 31] proved the same kind of result for semilocal endomorphisms rings of certain classes of torsion free abelian groups of finite rank. For further information, see the survey paper [29]. Our results give a new twist to the situation, as they indicate that when considering countable direct sums of such modules a rich supply of new direct summands might appear.

Let us mention a connection between projective modules over noetherian semilocal ring and integral representation theory. In [4 the so called generalized lattices were investigated. For a Dedekind domain $D$ with a quotient field $K$ we consider an order $R$ in a separable $K$-algebra. An $R$-module $M$ is said to be a generalized $R$-lattice provided it is projective as a $D$-module. If $M$ is also finitely generated, $M$ is a lattice over $R . R$ is said to be of finite lattice type if there exist only finitely many indecomposable lattices up to isomorphism. Suppose that $R$ is of finite lattice type and let $A$ be the direct sum of a representative set of isomorphism classes of indecomposable lattices. By [4], the category of generalized lattices over $R$ and the category of projective modules over $\operatorname{End}_{R}(A)$ are equivalent. For any maximal ideal $\mathcal{M}$ of $D$ let $R_{(\mathcal{M})}$ be the localization of $R$ in $D \backslash \mathcal{M}$ and let $R_{(0)}$ be the localization of $R$ in $D \backslash\{0\}$. Then monoid homomorphisms

$$
V^{*}\left(\operatorname{End}_{R}(A)\right) \rightarrow V^{*}\left(\operatorname{End}_{R_{(\mathcal{M})}}\left(A \otimes_{R} R_{(\mathcal{M})}\right)\right) \rightarrow V^{*}\left(\operatorname{End}_{R_{(0)}}\left(A \otimes_{R} R_{(0)}\right)\right)
$$


give approximations of generalized lattices over $R$ by projective modules over the noetherian semilocal rings $\operatorname{End}_{R_{(\mathcal{M})}}\left(A \otimes_{R} R_{(\mathcal{M})}\right)$ and an artinian $\operatorname{ring} \operatorname{End}_{R_{(0)}}\left(A \otimes_{R} R_{(0)}\right)$. For further results on generalized lattices see [26].

The paper is structured as follows, in $\$ 1$ we introduce the basic language used throughout the paper. We describe the monoids of projective modules, specializing to a semisimple artinian ring, we recall the results needed to understand the relation between these monoids when considered over $R$ and over $R / J(R)$ emphasizing on the particular case of semilocal rings. In $\$ 2$ we specialize to the noetherian case; we recall the results from [23] essential for our investigation and we state our main Theorem 2.7. Sections 3 , 4 and 5 deal with the realization part of the proof of Theorem 2.7 $\$ 3$ shows how to construct principal ideal domains with prescribed semisimple factor modulo the Jacobson radical, in 4 we provide all the results we need on ring pullbacks in order to be able to realized the monoids we want as $V^{*}(R)$ of semilocal noetherian algebras in $\$ 5$,

In section 6 we turn towards monoids. We recall some basics on full affine monoids, and we prove the auxiliary results that will allow us to conclude the proof of Theorem 2.7 in 97. In section 8 we mostly present some examples to illustrate consequences of Theorem 2.7 and to show the potential of the pullback constructions also outside the noetherian setting. For example, in 8.5 we construct a semilocal ring such that all projective left $R$-modules are free while $R$ has a nonzero (infinitely generated) right projective module that is not a generator, so it is not a direct sum of finitely generated projective modules. Such an example also shows that the notion of p-connected ring is not left-right symmetric; this answers in the negative a question in [15, p. 310]. Recall that, according to Bass [1, a ring is (left) $p$-connected if every nonzero left projective module is a generator

All rings have 1, ring morphisms and modules are unital. We shall usually consider right modules.

Our convention is $\mathbb{N}=\{1,2, \ldots\}$, and we denote the nonnegative integers by $\mathbb{N}_{0}=$ $\{0,1,2, \ldots\}$.

Another basic object in this paper is the monoid $\left(\mathbb{N}_{0}^{*},+, 0\right)$ whose underlying set is $\mathbb{N}_{0} \cup\{\infty\}$, the operation + is the extension of addition of non-negative integers by the rule $\infty+x=x+\infty=\infty$. Sometimes we will be also interested in extending the product of $\mathbb{N}_{0}$ to $\mathbb{N}_{0}^{*}$ by setting $\infty \cdot 0=0$ and $\infty \cdot n=\infty$ for any $n \in \mathbb{N}_{0}^{*} \backslash\{0\}$.

For any right $R$-module $M$ the trace of $M$ in $R$ is the two sided ideal of $R$

$$
\operatorname{Tr}_{R}(M)=\operatorname{Tr}(M)=\sum_{f \in \operatorname{Hom}_{R}(M, R)} f(M) .
$$

If $X \subseteq M$ then we denote the right annihilator of $X$ by

$$
r_{R}(X)=\{r \in R \mid m r=0 \text { for any } m \in X\} .
$$

If $N$ is a left $R$-module and $Y \subseteq N$ then we denote the left annihilator of $Y$ by

$$
l_{R}(Y)=\{r \in R \mid r n=0 \text { for any } n \in Y\} .
$$




\section{Monoids of projective modules}

We recall the following definitions

Definition 1.1 Let $(M,+, 0)$ be a commutative additive monoid. An element $x \in M$ is said to be an order unit or an archimedean element of $M$ if for any $y \in M$ there exists $n \in \mathbb{N}$ and $z \in M$ such that $n x=y+z$.

The monoid $M$ is said to be reduced if, for any $x \in M, x+y=0$ implies $x=0=y$.

Let $x, y \in M$. The relation $x \leq y$ if and only if there exists $z \in M$ such that $x+z=y$ is a preorder order on $M$ that is called the algebraic order or, more properly, the algebraic preorder.

For example, any $x \in \mathbb{N}_{0}^{*}$ satisfies that $x \leq \infty$. If $k \geq 1$ the algebraic preorder of $\mathbb{N}_{0}^{k}$ and in $\left(\mathbb{N}_{0}^{*}\right)^{k}$ is the component-wise order and it is a partial order.

Note that if $M$ is a monoid preordered with the algebraic preorder then all the elements must be positive.

Let $R$ be a ring. We denote by $V(R)$ the monoid of isomorphism classes of finitely generated projective right $R$-modules with the operation induced by the direct sum. This is to say, if $P_{1}$ and $P_{2}$ are finitely generated projective right $R$-modules then $\left\langle P_{1}\right\rangle+\left\langle P_{2}\right\rangle=$ $\left\langle P_{1} \oplus P_{2}\right\rangle$. The monoid $V(R)$ is commutative, reduced and it has an order unit $\langle R\rangle$. We usually think on $V(R)$ as a monoid preordered by the algebraic preorder.

Similarly, we define $V^{*}(R)$ to be the monoid of isomorphism classes of countably generated projective right $R$-modules with the sum induced by the direct sum. Clearly $V(R)$ is a preordered submonoid of the preordered monoid $V^{*}(R)$.

The functor $\operatorname{Hom}_{R}(-, R)$ induces a monoid isomorphism between $V(R)=V\left(R_{R}\right)$ and the monoid of isomorphism classes of finitely generated left projective modules $V\left({ }_{R} R\right)$. This is no longer true for countably generated projective modules so, in general, $V^{*}(R)=V^{*}\left(R_{R}\right)$ is not isomorphic to $V^{*}\left({ }_{R} R\right)$, cf. Example 8.5.

If $\varphi: R_{1} \rightarrow R_{2}$ is a ring morphism then the functor $-\otimes_{R_{1}} R_{2}$ induces a morphism of monoids with order unit $V(\varphi): V\left(R_{1}\right) \rightarrow V\left(R_{2}\right)$ and a morphism of monoids $V^{*}(\varphi): V^{*}\left(R_{1}\right) \rightarrow$ $V^{*}\left(R_{2}\right)$. Both morphisms are given by the formula $\langle P\rangle \mapsto\left\langle P \otimes_{R_{1}} R_{2}\right\rangle$. Another useful way to describe these monoid morphisms is describing projective modules via idempotent matrices.

Let $P_{R_{1}}$ be a finitely generated projective right $R_{1}$ modules. There exist $n \in \mathbb{N}$ and an idempotent matrix $E \in M_{n}\left(R_{1}\right)$ such that $P \cong E R_{1}^{n}$, then $V(\varphi)\left(\left\langle E R_{1}^{n}\right\rangle\right)=\left\langle M_{n}(\varphi)(E) R_{2}^{n}\right\rangle$ where $M_{n}(\varphi): M_{n}\left(R_{1}\right) \rightarrow M_{n}\left(R_{2}\right)$ is the map defined by $M_{n}(\varphi)\left(a_{i j}\right)=\left(\varphi\left(a_{i j}\right)\right)$. One proceeds similarly with the countably generated projective right $R_{1}$-modules taking instead of finite matrices elements in $\operatorname{CFM}\left(R_{1}\right)$ and $\operatorname{CFM}\left(R_{2}\right)$ the rings of (countable) column finite matrices with entries in $R_{1}$ and $R_{2}$ respectively.

\subsection{The semisimple artinian case}

Let $R$ be a semisimple artinian ring. Therefore, there exist $k \in \mathbb{N}, n_{1}, \ldots, n_{k} \in \mathbb{N}$, $D_{1}, \ldots, D_{k}$ division rings and an isomorphism $\varphi: R \rightarrow M_{n_{1}}\left(D_{1}\right) \times \cdots \times M_{n_{k}}\left(D_{k}\right)$ with kernel $J(R)$. 
Let $\left(V_{1}, \ldots, V_{k}\right)$ be an ordered set of representatives of the isomorphism classes of simple right $R$-modules such that $\operatorname{End}_{R}\left(V_{i}\right) \cong D_{i}$ and, hence, $\operatorname{dim}\left(D_{i} V_{i}\right)=n_{i}$ for $i=1, \ldots, k$. If $P_{R}$ is a finitely generated projective module then $P_{R} \cong V_{1}^{x_{1}} \oplus \cdots \oplus V_{k}^{x_{k}}$. The assignment $\langle P\rangle \mapsto\left(x_{1}, \ldots, x_{k}\right) \in \mathbb{N}_{0}^{k}$ induces an isomorphism of monoids $\operatorname{dim}_{\varphi}: V(R) \rightarrow \mathbb{N}_{0}^{k}$. Since $\operatorname{dim}_{\varphi}(\langle R\rangle)=\left(n_{1}, \ldots, n_{k}\right)$, taking $\left(n_{1}, \ldots, n_{k}\right)$ as the order unit of $\mathbb{N}_{0}^{k}, \operatorname{dim}_{\varphi}$ becomes an isomorphism of monoids with order unit. We call $\operatorname{dim}_{\varphi}(\langle P\rangle)$ or, by abuse of notation $\operatorname{dim}_{\varphi}(P)$, the dimension vector of the (finitely generated) projective module $P$.

The morphism $\operatorname{dim}_{\varphi}$ extends to a monoid morphism $\operatorname{dim}_{\varphi}: V^{*}(R) \rightarrow\left(\mathbb{N}_{0}^{*}\right)^{k}$ by setting $\operatorname{dim}_{\varphi}\left(\left\langle V_{i}^{(\omega)}\right\rangle\right)=\left(0, \ldots, \infty^{i)}, \ldots, 0\right)$ for $i=1, \ldots, k$. Again, we call $\operatorname{dim}_{\varphi}(\langle P\rangle)$ the dimension vector of the (countably generated) projective module $P$.

Throughout the paper, especially in sections 5 and 8 , it is important to keep in mind how to compute dimension vectors in terms of idempotent matrices. If $P$ is a finitely generated (countably generated) right projective module such that $\operatorname{dim}_{\varphi}(P)=\left(x_{1}, \ldots, x_{k}\right)$ then $P \cong$ $\left(E_{1}, \ldots, E_{k}\right) \cdot F$ where $F$ is a finitely generated (countably generated) free right $R$-module and $E_{i}$ are idempotent matrices over $M_{n_{i}}\left(D_{i}\right)\left(\right.$ over $\left.\operatorname{CFM}\left(D_{i}\right)\right)$ such that $\operatorname{rank}_{D_{i}}\left(E_{i}\right)=x_{i}$ for $i=1, \ldots, k$.

Finally, notice that $\operatorname{dim}_{\varphi}$ depends on the ordering of the isomorphism classes of the simple right modules. Therefore when we refer to a $\operatorname{dim}_{\varphi}$ function or to dimension vectors we implicitly assume that we have chosen an ordering of the simple modules. If we explicitly state that the semisimple artinian ring $R$ is isomorphic to $M_{n_{1}}\left(D_{1}\right) \times \cdots \times M_{n_{k}}\left(D_{k}\right)$, for $D_{1}, \ldots, D_{k}$ division rings, then we assume we are choosing an ordered family of representatives of the isomorphism classes of simple right (or left) $R$-modules $\left(V_{1}, \ldots, V_{k}\right)$ such that $\operatorname{End}_{R}\left(V_{i}\right) \cong D_{i}$ for $i=1, \ldots, k$.

To easy the work with the elements in $\mathbb{N}_{0}^{*}$ we shall use the following definitions.

Definition 1.2 Let $\mathbf{x}=\left(x_{1}, \ldots, x_{k}\right) \in\left(\mathbb{N}_{0}^{*}\right)^{k}$. We define

$$
\operatorname{supp}(\mathbf{x})=\left\{i \in\{1, \ldots, k\} \mid x_{i} \neq 0\right\}
$$

and we refer to this set as the support of $\mathbf{x}$. We also define

$$
\inf -\operatorname{supp}(\mathbf{x})=\left\{i \in\{1, \ldots, k\} \mid x_{i}=\infty\right\},
$$

we refer to this set as the infinite support of $\mathbf{x}$.

\subsection{Passing modulo the Jacobson radical}

First we recall the following well known Lemma

Lemma 1.3 Let $R$ be a ring with Jacobson radical $J(R)$. Let $P$ and $Q$ be projective right $R$-modules.

(i) Assume $P$ and $Q$ are finitely generated. If there exists a projective right $R / J(R)$ module $X$ such that $P / P J(R) \cong Q / Q J(R) \oplus X$ then there exists a projective right $R$-module $Q^{\prime}$ such that $P \cong Q \oplus Q^{\prime}$ and $Q^{\prime} / Q^{\prime} J(R) \cong X$. 
(ii) Assume only that $Q$ is finitely generated. If $f: P / P J(R) \rightarrow Q / Q J(R)$ is an onto module homomorphism then $Q$ is isomorphic to a direct summand of $P$.

If, in the above Lemma, neither $P$ nor $Q$ are finitely generated then even the weaker divisibility property (ii) is lost. It was shown in 22 that it is still true that projective modules isomorphic modulo the Jacobson radical are isomorphic. We recall this fundamental result in the next statement together with a weaker property on lifting pure monomorphisms.

We recall that a right module monomorphism $f: M_{1} \rightarrow M_{2}$ is said to be a pure monomorphism if, for any left module $N, f \otimes_{R} N: M_{1} \otimes_{R} N \rightarrow M_{2} \otimes_{R} N$ remains a monomorphism. For example, if $f$ is a (locally) split monomorphism then it is pure. If $f$ is a monomorphism between two projective modules then $f$ is pure if and only if coker $f$ is a flat module if and only if $f$ is locally split.

Theorem 1.4 Let $R$ be any ring, and let $P$ and $Q$ be projective right $R$-modules.

(i) [13, Proposition 6.1] A module homomorphism $f: P \rightarrow Q$ is a pure monomorphism if and only if so is the induced map $\bar{f}: P / P J(R) \rightarrow Q / Q J(R)$.

(ii) [22, Theorem 2.3] If $f: P / P J(R) \rightarrow Q / Q J(R)$ is an isomorphism of right $R / J(R)$ modules then there exists an isomorphism of right $R$-modules $g: P \rightarrow Q$ such that the induced morphism $\bar{g}: P / P J(R) \rightarrow Q / Q J(R)$ coincides with $f$.

Theorem 1.4(ii) allows us to see the monoids of projective modules over a ring $R$ as submonoids of the monoids of projective modules over $R / J(R)$. To give the assertion in a more precise way we shall use the following notion (cf. [11]).

Definition 1.5 $A$ submonoid $A$ of a monoid $C$ is said to be a full submonoid of $C$ if for any $x \in A$ and any $t \in C, x+t \in A$ implies $t \in A$. If $f: A \rightarrow C$ is an injective monoid homomorphism and $\operatorname{Im}(f)$ is a full submonoid of $C$ we say that $f$ is a full embedding.

$A$ full affine monoid is a full submonoid of a finitely generated free commutative monoid, and $a$ full affine embedding is a full embedding into a finitely generated free commutative monoid.

See Proposition 6.2 for a characterization of full affine submonoids of $\mathbb{N}_{0}^{k}$.

We note that in the terminology of 2] a full affine embedding is a pure embedding of monoids.

Corollary 1.6 Let $R$ be a ring with Jacobson radical $J(R)$, and let $\pi: R \rightarrow R / J(R)$ denote the canonical projection. Then:

(i) $V(\pi): V(R) \rightarrow V(R / J(R))$ is a full embedding of monoids with order unit. In particular, the algebraic preorder on $V(\pi)(V(R))$ coincides with the one induced by the algebraic preorder on $V(R / J(R))$.

(ii) $V^{*}(\pi): V^{*}(R) \rightarrow V^{*}(R / J(R))$ is an injective monoid morphism.

There is an interesting intermediate submonoid between $V(R)$ and $V^{*}(R)$. 
Definition 1.7 Let $R$ be a ring. Set $W(R)=W\left(R_{R}\right)$ to be the additive monoid of isomorphism classes of projective right $R$-modules that are pure submodules of a finitely generated free right $R$-module. The addition on $W(R)$ is induced by the direct sum of modules.

Analogously, $W\left({ }_{R} R\right)$ is the additive monoid of isomorphism classes of projective left $R$-modules that are pure submodules of a finitely generated free right $R$-module.

For example, if $R=\mathcal{C}([0,1])$ is the ring of real valued continuous functions defined on the interval $[0,1]$ then the ideal

$$
I=\{f \in R \mid \text { there exists } \varepsilon>0 \text { such that } f([0, \varepsilon])=0\}
$$

is countably generated, projective and pure inside $R$. Therefore $\langle I\rangle \in W(R) \backslash V(R)$.

The notation $W(R)$ is borrowed from the $C^{*}$-algebra world, as we think on $W(R)$ as the discrete analogue of the Cuntz monoid (cf. [7])

The following result, which is a consequence of [13, Theorem 7.1] and Theorem 1.4, describes one way to obtain elements in $W(R) \backslash V(R)$ and which is the only one when the ring $R$ is semilocal.

Proposition 1.8 Fix $n \in \mathbb{N}$. Let $R$ be a ring. Let $P_{1}, P_{2}$ be finitely generated projective right $R / J(R)$ modules such that $(R / J(R))^{n} \cong P_{1} \oplus P_{2}$. Then the following statements are equivalent

(i) There exists a projective right $R$-module $P$ such that $P / P J(R) \cong P_{1}$.

(ii) There exists a pure right submodule $M$ of $R^{n}$ such that $M / M J(R) \cong P_{1}$.

(iii) There exists a projective left $R$-module $Q$ such that $Q / J(R) Q \cong \operatorname{Hom}_{R / J(R)}\left(P_{2}, R / J(R)\right)$.

In this case $P$ and $Q$ are countably generated pure submodules of $R^{n}$. They are finitely generated if and only if there exists a projective right $R$-module $P^{\prime}$ such that $P^{\prime} / P^{\prime} J(R) \cong$ $P_{2}$.

Observe that, by Theorem 1.4, the isomorphism class of the module $P$ appearing in Proposition 1.8 is an element of $W\left(R_{R}\right)$ and the isomorphism class of $Q$ gives an element of $W\left({ }_{R} R\right)$. Therefore if $P$ is not finitely generated, $\langle P / P J(R)\rangle \leq\left\langle(R / J(R))^{n}\right\rangle$ in $W(R / J(R))$ but $P$ is not a direct summand of $R^{n}$, so that $\langle P\rangle$ and $\left\langle R^{n}\right\rangle$ are uncomparable in $W(R)$. So that, in general, the algebraic preorder on $W(R)$ does not coincide with the order induced by the algebraic preorder on $W(R / J(R))$.

We will go back to the monoid $W(R)$ in 8 , as we are mainly interested in noetherian rings and then, clearly, $V(R)=W(R)$. Results of Lazard [20] show that this also holds just assuming ascending chain condition on annihilators.

Proposition 1.9 Let $R$ be a ring such that, for any $n \in \mathbb{N}, M_{n}(R)$ has the ascending chain condition on right annihilators of elements. Then a pure submodule of a finitely generated free right $R$-module is finitely generated.

Proof. Combine [20, Lemme 2(i)] with the argument in [12, Corollary 3.6]. 


\section{$1.3 \quad$ Semilocal rings}

Let $R$ be a semilocal ring such that $R / J(R) \cong M_{n_{1}}\left(D_{1}\right) \times \cdots \times M_{n_{k}}\left(D_{k}\right)$ for suitable division rings $D_{1}, \ldots, D_{k}$. Fix an onto ring homomorphism $\varphi: R \rightarrow M_{n_{1}}\left(D_{1}\right) \times \cdots \times M_{n_{k}}\left(D_{k}\right)$ such that $\operatorname{Ker} \varphi=J(R)$. Then there is an induced ring isomorphism $\bar{\varphi}: R / J(R) \rightarrow M_{n_{1}}\left(D_{1}\right) \times$ $\cdots \times M_{n_{k}}\left(D_{k}\right)$, so that we have a dimension function $\operatorname{dim}_{\bar{\varphi}}$, cf. $\S 1.1$ For any countably generated projective right $R$-module $P$, set

$$
\operatorname{dim}_{\varphi}(\langle P\rangle):=\operatorname{dim}_{\bar{\varphi}}\left(\left\langle P \otimes_{R} R / J(R)\right\rangle\right)=\operatorname{dim}_{\bar{\varphi}}(\langle P / P J(R)\rangle) .
$$

By Corollary 1.6, $\operatorname{dim}_{\varphi}(V(R))$ is a full affine submonoid of $\mathbb{N}_{0}^{k}$ with order unit $\left(n_{1}, \ldots, n_{k}\right)$ and $\operatorname{dim}_{\varphi}\left(V^{*}(R)\right)$ is a submonoid of $\left(\mathbb{N}_{0}^{*}\right)^{k}$. For some considerations on the order induced on $V^{*}(R)$ by $V^{*}(R / J(R))$ we refer to Corollary 2.5 and to $\S 8$

It was shown in [10] that the full affine property characterizes the monoids $A$ with order unit that can be realized as $V(R)$ of some semilocal ring $R$. More precisely, if $A$ is a full affine submonoid of $\mathbb{N}_{0}^{k}$ with order unit $\left(n_{1}, \ldots, n_{k}\right)$ then there exist a semilocal hereditary ring $R$, $D_{1}, \ldots, D_{k}$ division rings and an onto ring homomorphism $\varphi: R \rightarrow M_{n_{1}}\left(D_{1}\right) \times \cdots \times M_{n_{k}}\left(D_{k}\right)$ with kernel $J(R)$ such that $\operatorname{dim}_{\varphi} V(R)=A$.

Note that since over a hereditary ring any projective module is a direct sum of finitely generated projective modules, it follows that for a hereditary ring $R$ as above

$$
\operatorname{dim}_{\varphi} V^{*}(R)=A+\infty \cdot A \subseteq\left(\mathbb{N}_{0}^{*}\right)^{k}
$$

see Corollary 8.1 and Proposition 6.7

\section{Semilocal rings: The noetherian case}

We start this section recalling some results on projective modules over noetherian semilocal rings from 23] and adapting them to our purposes. We also state in 2.7 our main characterization theorem.

It is well known that the trace ideal of a projective module is an idempotent ideal. Whitehead in 24] characterized idempotent ideals that are trace ideals of countably generated projective modules. His results yield that in a noetherian ring any idempotent ideal is a trace ideal of a countably generated projective module. In [23, Př́hoda noted that Whitehead's ideas can be extended to prove that if $I$ is an idempotent ideal of a noetherian ring $R$ then any finitely generated projective $R / I$-module can be extended to a projective $R$-module. For further quotation we state these results.

Proposition 2.1 Let $R$ be a noetherian ring. Then the following statements are equivalent for a two sided ideal $I$

(i) $I^{2}=I$.

(ii) There exists a countably generated projective right $R$-module $P$ such that $\operatorname{Tr}(P)=I$.

(iii) For any finitely generated projective right $R / I$-module $P^{\prime}$ there exists a countably generated projective right $R$-module $P$ such that $P / P I \cong P^{\prime}$ and $I \subseteq \operatorname{Tr}(P)$. 
(iv) There exists a countably generated projective left $R$-module $Q$ such that $\operatorname{Tr}(Q)=I$.

(v) For any finitely generated projective left $R / I$-module $Q^{\prime}$ there exists a countably generated projective left $R$-module $Q$ such that $Q / I Q \cong Q^{\prime}$ and $I \subseteq \operatorname{Tr}(Q)$.

Proof. Combine [24, Corollary 2.7] with [23, Lemma 2.6].

Trace ideals of projective modules keep memory of the semisimple factors of the projective module.

Lemma 2.2 Let $R$ be a semilocal ring, and let $P$ be a projective right module with trace ideal I. Let $V_{R}$ be a simple right $R$-module with endomorphism ring $D$, and let $W=$ $\operatorname{Hom}_{D}(V, D) \cong \operatorname{Hom}_{R}(V, R / J(R))$ be its dual simple left $R$-module. Then the following statement are equivalent:

(i) $V$ is a quotient of $P$.

(ii) $V$ is a quotient of $I$.

(iii) $I+r_{R}(V)=I+l_{R}(W)=R$.

(iv) $W$ is a quotient of $I$.

In particular, if $I$ is also the trace ideal of a left projective module $Q$ then the above statements are also equivalent to the fact that $W$ is a quotient of $Q$.

Proof. The equivalence of (i) and (ii) is a particular case of [23, Lemma 3.3]. It is clear that (iii) is equivalent to (ii) because, for a semilocal ring, $r_{R}(V)=l_{R}(W)$ is a maximal two-sided ideal of $R$. Statements (iii) and (iv) are equivalent by the symmetry of (iii).

Theorem 2.3 [23] Let $R$ be a noetherian semilocal ring. Let $V_{1}, \ldots, V_{k}$ be an ordered set of representatives of the isomorphism classes of simple right $R$-modules.

For $i=1, \ldots, k$, let $D_{i}=\operatorname{End}_{R}\left(V_{i}\right)$ and $W_{i}=\operatorname{Hom}_{D_{i}}\left(V_{i}, D_{i}\right) \cong \operatorname{Hom}_{R}\left(V_{i}, R / J(R)\right)$. So that $W_{1}, \ldots, W_{k}$ is an ordered set of representatives of the isomorphism classes of simple left $R$-modules. Let $S$ be a subset of $\{1, \ldots, k\}$. Assume that there exists a countably generated projective right $R$ module $P$ such that

$$
P / P J(R) \cong\left(\oplus_{i \in\{1, \ldots, k\} \backslash S} V_{i}^{n_{i}}\right) \oplus\left(\oplus_{i \in S} V_{i}^{(\omega)}\right),
$$

where $n_{i} \in \mathbb{N}_{0}$. Then the following statements hold:

(1) There exists a countably generated projective right $R$-module $P^{\prime}$ such that $P^{\prime} / P^{\prime} J(R) \cong$ $\oplus_{i \in S} V_{i}^{(\omega)}$. Hence $P \cong P \oplus P^{\prime}$.

(2) Let $I$ be the trace ideal of $P^{\prime}$. Then $P / P I$ is a finitely generated right $R / I$-module such that

$$
P / P I \otimes_{R / I}(R / I) / J(R / I) \cong P / P(I+J(R)) \cong \oplus_{i \in\{1, \ldots, k\} \backslash S} V_{i}^{n_{i}} .
$$


(3) There exists a countably generated projective left $R$-module $Q$ such that

$$
Q / J(R) Q \cong\left(\oplus_{i \in\{1, \ldots, k\} \backslash S} W_{i}^{n_{i}}\right) \oplus\left(\oplus_{i \in S} W_{i}^{(\omega)}\right)
$$

(4) There exists a countably generated projective left $R$-module $Q^{\prime}$ such that $Q^{\prime} / J(R) Q^{\prime} \cong$ $\oplus_{i \in S} W_{i}^{(\omega)}$. Hence $Q \cong Q \oplus Q^{\prime}$.

Therefore, $V^{*}\left(R_{R}\right) \cong V^{*}\left({ }_{R} R\right)$ and, fixing $\varphi: R \rightarrow M_{n_{1}}\left(D_{1}\right) \times \cdots \times M_{n_{k}}\left(D_{k}\right)$ an onto ring homomorphism with kernel $J(R)$, we obtain that $\operatorname{dim}_{\varphi} V^{*}\left(R_{R}\right)=\operatorname{dim}_{\varphi} V^{*}\left({ }_{R} R\right)$ and that $\operatorname{dim}_{\varphi} V(R)=\left(\operatorname{dim}_{\varphi} V^{*}(R)\right) \cap \mathbb{N}_{0}^{k}$.

Proof. (1). The existence of $P^{\prime}$ follows from [23, Proposition 3.4]. The isomorphism $P \cong P \oplus P^{\prime}$ follows from Theorem 1.4(ii).

Statement (2) is also part of [23, Proposition 3.4].

By Proposition 2.1 $I$ is also the trace ideal of a projective left $R$-module $M$. As $M^{(\omega)} / J(R) M^{(\omega)}$ is semisimple and contains all semisimple factors of $M$ it follows from Lemma 2.2 that $M^{(\omega)} / J(R) M^{(\omega)} \cong \oplus_{i \in S}\left(V_{i}^{*}\right)^{(\omega)}$. Therefore taking $Q^{\prime}=M^{(\omega)}$ we deduce that the first statement of (4) holds.

By (2), $P / P I$ is a finitely generated $R / I$-module. Therefore $\bar{Q}=\operatorname{Hom}_{R / I}(P / P I, R / I)$ is a finitely generated projective left $R / I$-module such that $\bar{Q} / J(\bar{Q}) \cong \oplus_{i \in\{1, \ldots, k\} \backslash S} W_{i}^{n_{i}}$. By Proposition 2.1, there exists a projective left $R$-module $Q_{1}$ such that $Q_{1} / I Q_{1} \cong \bar{Q}$. Then $Q=Q_{1} \oplus Q^{\prime}$ fulfills the requirements of statement (3) and the second half of statement (4).

Finally, note that the assignment $\langle P\rangle \mapsto\langle Q\rangle$ induces an isomorphism between $V^{*}\left(R_{R}\right)$ and $V^{*}\left({ }_{R} R\right)$ such that $\operatorname{dim}_{\varphi}\left(V^{*}\left(R_{R}\right)\right)=\operatorname{dim}_{\varphi}\left(V^{*}\left({ }_{R} R\right)\right)$. The claim on $\operatorname{dim}_{\varphi} V(R)$ follows either from (2) or from combining Proposition [1.8 with Proposition [1.9]

In Example 8.5 we will see that the isomorphism between $V^{*}\left({ }_{R} R\right)$ and $V^{*}\left(R_{R}\right)$ does not exist for general semilocal rings.

As a corollary of Theorem 2.3 we note that, in the context of noetherian semilocal rings, the divisibility property of Lemma 1.3(ii) still holds for general projective modules.

Corollary 2.4 Let $R$ be a noetherian semilocal ring. Let $P$ and $Q$ be projective right $R$ modules such that $P / P J(R)$ is isomorphic to a direct summand of $Q / Q J(R)$ then $P$ is isomorphic to a direct summand of $Q$.

Proof. Since any projective module is a sum of countably generated projective modules we may assume that $P$ and $Q$ are countably generated [9, Proposition 2.50].

Let $V_{1}, \ldots, V_{k}$ be an ordered set of representatives of the isomorphism classes of simple right $R$-modules. Since $P / P J(R)$ is a homomorphic image of $Q / Q J(R)$ there exist $S^{\prime} \subseteq$ $S \subseteq\{1, \ldots, k\}$ such that

$$
Q / Q J(R) \cong\left(\oplus_{i \in\{1, \ldots, k\} \backslash S} V_{i}^{n_{i}}\right) \oplus\left(\oplus_{i \in S} V_{i}^{(\omega)}\right)
$$

and

$$
P / P J(R) \cong\left(\oplus_{i \in\{1, \ldots, k\} \backslash S^{\prime}} V_{i}^{m_{i}}\right) \oplus\left(\oplus_{i \in S^{\prime}} V_{i}^{(\omega)}\right)
$$


where $n_{i}$ and $m_{j}$ are in $\mathbb{N}_{0}$, and $n_{i}-m_{i} \in \mathbb{N}_{0}$ for any $i \in\{1, \ldots, k\} \backslash S$.

By Theorem [2.3, there exists a countably generated projective module $Q^{\prime}$ such that $Q^{\prime} / Q^{\prime} J(R) \cong \oplus_{i \in S} V_{i}^{(\omega)}$. Let $I$ be the trace ideal of $Q^{\prime}$. Again by Theorem 2.3, $\bar{P}=P / P I$ and $\bar{Q}=Q / Q I$ are finitely generated projective right $R / I$-modules.

Now

$$
\bar{Q} / \bar{Q} J(R / I) \cong Q / Q(I+J(R)) \cong \oplus_{i \in\{1, \ldots, k\} \backslash S} V_{i}^{n_{i}}
$$

and

$$
\bar{P} / \bar{P} J(R / I) \cong P / P(I+J(R)) \cong \oplus_{i \in\{1, \ldots, k\} \backslash S} V_{i}^{m_{i}}
$$

By Corollary 1.6 there exist a finitely generated projective right $R / I$-module $\bar{X}$ such that $\bar{X} / \bar{X} J(R / I) \cong \oplus_{i \in\{1, \ldots, k\} \backslash S} V_{i}^{n_{i}-m_{i}}$. By Proposition 2.1, there exists a countably generated projective right $R$-module $X$ such that $X / X I \cong \bar{X}$. By Theorem 1.4 (ii), $Q \cong P \oplus Q^{\prime} \oplus X$.

An alternative way to state Corollary 2.4 is thinking on $V^{*}(R)$ as a monoid with the partial order induced by the algebraic order of $V^{*}(R / J(R))$.

Corollary 2.5 Let $R$ be a semilocal ring. Consider the following relation over $V^{*}(R)$, $\langle P\rangle \leq\langle Q\rangle$ if and only if $P / P J(R)$ is isomorphic to a direct summand of $Q / Q J(R)$. Then

(i) $\langle P\rangle \leq\langle Q\rangle$ if and only if there exists a pure embedding $f: P \rightarrow Q$.

(ii) $\leq$ is a partial order relation that refines the algebraic order on $V^{*}(R)$.

(iii) If, in addition, $R$ is noetherian then the partial order induced by $\leq$ over $V^{*}(R)$ is the algebraic order.

Proof. (i). If $\langle P\rangle \leq\langle Q\rangle$ then there exists a splitting monomorphism $\bar{f}: P / P J(R) \rightarrow$ $Q / Q J(R)$ which by Theorem 1.4(i) lifts to a pure monomorphism $f: P \rightarrow Q$. Conversely, if $f: P \rightarrow Q$ is a pure monomorphism of right $R$-modules then the induced map $f \otimes_{R}$ $R / J(R): P \otimes_{R} R / J(R) \rightarrow Q \otimes_{R} R / J(R)$ is a pure monomorphism of $R / J(R)$-modules. Since $R / J(R)$ is semisimple, $f \otimes_{R} R / J(R)$ is a split monomorphism.

(ii). It is clear that $\leq$ is reflexive and transitive. As it is already observed in [22, Theorem 1.4 implies that $\leq$ is also antisymmetric.

If $P$ is isomorphic to a direct summand of $Q$, then $P / P J(R)$ is also isomorphic to a direct summand of $Q / Q J(R)$. Hence $\langle P\rangle \leq\langle Q\rangle$, that is, $\leq$ refines the algebraic order on $V^{*}(R)$.

(iii). It is a consequence of Corollary 2.4.

After some amount of work, it will turn out that Proposition 2.1 and Theorem 2.3 contain all the information needed to describe $V^{*}(R)$ for $R$ a noetherian semilocal ring.

Definition 2.6 Let $k \geq 1$. A submonoid $M$ of $\left(\mathbb{N}_{0}^{*}\right)^{k}$ is said to be a monoid defined by a system of equations provided that there exist $D \in M_{n \times k}\left(\mathbb{N}_{0}\right), E_{1}, E_{2} \in M_{\ell \times k}\left(\mathbb{N}_{0}\right)$ and 
$m_{1}, \ldots, m_{n} \in \mathbb{N}, m_{i} \geq 2$ for any $i \in\{1, \ldots, n\}$, such that $M$ is the set of solutions in $\left(\mathbb{N}_{0}^{*}\right)^{k}$ of the system of equations

$$
D\left(\begin{array}{c}
t_{1} \\
\vdots \\
t_{k}
\end{array}\right) \in\left(\begin{array}{c}
m_{1} \mathbb{N}_{0}^{*} \\
\vdots \\
m_{n} \mathbb{N}_{0}^{*}
\end{array}\right) \quad(*) \quad \text { and } \quad E_{1}\left(\begin{array}{c}
t_{1} \\
\vdots \\
t_{k}
\end{array}\right)=E_{2}\left(\begin{array}{c}
t_{1} \\
\vdots \\
t_{k}
\end{array}\right)
$$

where $\ell, n \geq 0$. By convention, $\ell$ or $n$ equal to zero means that either $(*)$ or $(* *)$ are empty systems.

As we shall recall in Proposition 6.2. any full affine monoid of $\mathbb{N}_{0}^{k}$ is of the form $M \cap \mathbb{N}_{0}^{k}$ where $M$ is a submonoid of $\left(\mathbb{N}_{0}^{*}\right)^{k}$ defined by a system of equations.

Now we can state our main theorem,

Theorem 2.7 Let $k \in \mathbb{N}$. Let $M$ be a submonoid of $\left(\mathbb{N}_{0}^{*}\right)^{k}$ containing $\left(n_{1}, \ldots, n_{k}\right) \in \mathbb{N}^{k}$. Then the following statements are equivalent:

(1) $M$ is defined by a system of equations.

(2) For any field $F$ there exist a noetherian semilocal $F$-algebra $R$, a semisimple $F$-algebra $S=M_{n_{1}}\left(D_{1}\right) \times \cdots \times M_{n_{k}}\left(D_{k}\right)$, where $D_{1}, \ldots, D_{k}$ are division rings, and an onto morphism of F-algebras $\varphi: R \rightarrow S$ with $\operatorname{Ker} \varphi=J(R)$ such that $\operatorname{dim}_{\varphi} V^{*}(R)=M$. In particular, $\operatorname{dim}_{\varphi} V(R)=M \cap \mathbb{N}_{0}^{k}$.

(3) There exist a noetherian semilocal ring $R$, a semisimple ring $S=M_{n_{1}}\left(D_{1}\right) \times \cdots \times$ $M_{n_{k}}\left(D_{k}\right)$, were $D_{1}, \ldots, D_{k}$ are division rings, and an onto ring morphism $\varphi: R \rightarrow S$ with $\operatorname{Ker} \varphi=J(R)$ such that $\operatorname{dim}_{\varphi} V^{*}(R)=M$. Therefore, $\operatorname{dim}_{\varphi} V(R)=M \cap \mathbb{N}_{0}^{k}$.

Remark 2.8 We follow the notation of Definition [2.6. As it is done for full affine monoids in [3, Exercise 6.4.16] or [2, Proof of Theorem 2.29], if $M \subseteq\left(\mathbb{N}_{0}^{*}\right)^{k}$ is defined by a system of equations as in 2.6 then it is isomorphic to the submonoid $M^{\prime}$ of $\left(\mathbb{N}_{0}^{*}\right)^{k+n}$ defined by system of linear diophantine equalities

$$
D\left(\begin{array}{c}
t_{1} \\
\vdots \\
t_{k}
\end{array}\right)=\left(\begin{array}{ccc}
m_{1} & \cdots & 0 \\
\vdots & \ddots & \vdots \\
0 & \cdots & m_{n}
\end{array}\right)\left(\begin{array}{c}
t_{k+1} \\
\vdots \\
t_{k+n}
\end{array}\right) \quad \text { and } \quad E_{1}\left(\begin{array}{c}
t_{1} \\
\vdots \\
t_{k}
\end{array}\right)=E_{2}\left(\begin{array}{c}
t_{1} \\
\vdots \\
t_{k}
\end{array}\right)
$$

The isomorphism is given by the assignment

$$
\left(x_{1}, \ldots, x_{k}\right) \mapsto\left(x_{1}, \ldots, x_{k}, \frac{1}{m_{1}} \sum_{i=1}^{k} d_{1 j} x_{j}, \ldots, \frac{1}{m_{n}} \sum_{i=1}^{k} d_{n j} x_{j}\right),
$$

where we make the convention $\frac{\infty}{m_{i}}=\infty$.

Therefore it is important to take into account that we are considering our monoids always inside some fixed $\left(\mathbb{N}_{0}^{*}\right)^{k}$ or, in the ring context, that we are viewing $V^{*}(R)$ as a submonoid of $V^{*}(R / J(R))$. 
The monoid $\mathbb{N}_{0}^{*}$ is not cancellative, therefore the solutions of two systems of equations may coincide over $\mathbb{N}_{0}$ but be different when considered over $\mathbb{N}_{0}^{*}$. We illustrate this phenomena with an easy example.

Example 2.9 The set of solutions of the equation $x=y$ in $\mathbb{N}_{0}^{2}$ is $M=\left\{(n, n) \mid n \in \mathbb{N}_{0}\right\}$, and the set of solutions in $\left(\mathbb{N}_{0}^{*}\right)^{2}$ is $M+\infty \cdot M=M \cup\{(\infty, \infty)\}$.

The set of solutions of $2 x=x+y$ in $\mathbb{N}_{0}^{2}$ is, of course, also $M$ but in $\left(\mathbb{N}_{0}^{*}\right)^{2}$ is $M_{1}=$ $M \cup\left\{(\infty, n) \mid n \in \mathbb{N}_{0}^{*}\right\}$.

Finally, the set of solutions of $2 x+y=x+2 y$ in $\left(\mathbb{N}_{0}^{*}\right)^{2}$ is $M_{1} \cup\left\{(n, \infty) \mid n \in \mathbb{N}_{0}^{*}\right\}$.

Theorem 2.7 shows that, for noetherian semilocal rings, the description of $V^{*}(R)$ viewed inside $V^{*}(R / J(R))$ nicely extends the one of $V(R)$ inside $V(R / J(R))$ (cf. Proposition 6.2). In 80 we give examples showing that the picture for general semilocal rings must be more complicated.

\section{Semilocal principal ideal domains}

We recall that a ring $R$ is a principal ideal domain if $R$ is a right and left principal ideal domain, that is, if every right ideal of $R$ has the form $a R$ for some $a \in R$ and every left ideal of $R$ has the form $R a$ for some $a \in R$.

Semilocal principal ideal domains are a source of semilocal noetherian rings such that all projective modules are free. Our aim in this section is to construct semilocal PID's with certain types of semisimple factors.

Let $R$ be a commutative ring. Let $k \geq 1$, and let $\mathcal{M}_{1}, \ldots, \mathcal{M}_{k}$ be different maximal ideals of $R$. The localization of $R$ at the set $\Sigma=R \backslash\left(\mathcal{M}_{1} \cup \cdots \cup \mathcal{M}_{k}\right)$ is a semilocal ring such that modulo its Jacobson radical is isomorphic to $R / \mathcal{M}_{1} \times \cdots \times R / \mathcal{M}_{k}$.

Fuller and Shutters observed in [15] that the same procedure to construct semilocal rings can be extended to, non necessarily commutative, principal ideal domains by using Ore localization.

Proposition 3.1 [15, Proposition 4] Let $\varphi: R \rightarrow S$ be a surjective ring homomorphism of a principal ideal domain $R$ onto a semisimple artinian ring $S$. Let $\Sigma=\{a \in R \mid$ $\varphi(a)$ is invertible in $S\}$. Then:

(i) $\Sigma$ is a right and left Ore set.

(ii) The Ore localization $R_{\Sigma}$ of $R$ with respect to $\Sigma$ is a semilocal principal ideal domain, and the extension $\bar{\varphi}: R_{\Sigma} \rightarrow S$ of $\varphi$ induces an isomorphism $R_{\Sigma} / J\left(R_{\Sigma}\right) \cong S$.

Next result gives a source of examples where to apply Proposition 3.1.

Let $E$ be any ring, and let $\alpha: E \rightarrow E$ a (unital) ring morphism. The skew polynomial ring or the twisted polynomial ring is the ring

$$
E[x ; \alpha]=\left\{p(x)=p_{0} x^{m}+\cdots+p_{m} \mid m \in \mathbb{N}_{0} \text { and } p_{i} \in E \text { for } i=0, \ldots, m\right\}
$$


with componentwise addition and multiplication induced by the rule $x r=\alpha(r) x$ for any $r \in E$.

It is well known that if $E$ is a division ring and $\alpha$ is an automorphism then $E[x ; \alpha]$ has a right and a left division algorithm, hence, it is a principal ideal domain.

Proposition 3.2 Let $E$ be a field. Let $\alpha: E \rightarrow E$ be a field automorphism of order $n$ with fixed field $E^{\alpha}=\{a \in E \mid \alpha(a)=a\}$. Then the skew polynomial ring $R=E[x ; \alpha]$ has a simple factor isomorphic to $M_{n}\left(E^{\alpha}\right)$.

Moreover, if $E$ is infinite then, for any $k \in \mathbb{N}, R$ has a factor isomorphic to $M_{n}\left(E^{\alpha}\right)^{k}$.

Proof. We may assume that $n>1$.

Note the following fact that will be useful throughout the proof:

$\left(^{*}\right)$ Let $p(x)=x^{m}+p_{1} x^{m-1}+\cdots+p_{m} \in R$ be such that $p_{m} \neq 0$. If $a \in E$ satisfies that ap $(x) \in p(x) R$ then $\alpha^{m}(a)=a$.

As $\alpha$ has order $n$, the center of $R$ contains (in fact coincides with) $E^{\alpha}\left[x^{n}\right]$. Therefore, for any $0 \neq t \in E^{\alpha}$, the right ideal $\left(x^{n}-t\right) R$ is two-sided. As $R$ is a right principal ideal domain, $\left(^{*}\right)$ yields that $\left(x^{n}-t\right) R$ is a maximal two-sided ideal, so that $R /\left(x^{n}-t\right) R$ is a simple artinian ring. We claim that if $t=r^{n}$ for some $r \in E^{\alpha}$ then $R /\left(x^{n}-t\right) R \cong M_{n}\left(E^{\alpha}\right)$. To prove this we need to find a simple right $R /\left(x^{n}-t\right) R$-module such that its endomorphism ring is $E^{\alpha}$ and its dimension over $E^{\alpha}$ is $n$.

In $E^{\alpha}[x] \subseteq R$ we have a decomposition $x^{n}-r^{n}=(x-r) q(x)$. As $x^{n}-r^{n}$ is central in $R, V=R /(x-r) R$ is a right $R /\left(x^{n}-r^{n}\right) R$-module. It is readily checked that $V$ is a right $E$-vector space of dimension 1 , therefore it is a simple right $R /\left(x^{n}-r^{n}\right) R$-module.

$\operatorname{End}_{R}(V)=\mathcal{I} /(x-r) R$, where $\mathcal{I}=\{p(x) \in R \mid p(x)(x-r) \in(x-r) R\}$ is the idealizer of $(x-r) R$ in $R$. As any $p(x) \in R$ can be written in a unique way as $a+(x-r) q(x)$ for $a \in E$,

$$
\operatorname{End}_{R}(V) \cong \mathcal{I} \cap E=\{a \in E \mid a(x-r) \in(x-r) R\}
$$

By $\left(^{*}\right), \mathcal{I} \cap E=E^{\alpha}$ and, hence, $\operatorname{End}_{R}(V) \cong E^{\alpha}$. Since $E^{\alpha} V \cong E^{\alpha} E$ and, by Artin's Theorem, $\left[E: E^{\alpha}\right]=n$ we deduce that the dimension of $V$ over its endomorphism ring is $n$ as desired.

Now assume that $E$, and hence $E^{\alpha}$, is infinite. Fix $k \in \mathbb{N}$. Let $r_{1}, \ldots, r_{k} \in E^{\alpha}$ be such that $r_{1}^{n}, \ldots, r_{k}^{n}$ are $k$ different elements. Consider the ring homomorphism

$$
\Phi: R \rightarrow R /\left(x^{n}-r_{1}^{n}\right) R \times \cdots \times R /\left(x^{n}-r_{k}^{n}\right) R
$$

defined by $\Phi(p(x))=\left(p(x)+\left(x^{n}-r_{1}^{n}\right) R, \ldots, p(x)+\left(x^{n}-r_{k}^{n}\right) R\right)$. Clearly, Ker $\Phi=\cap_{i=1}^{k}\left(x^{n}-\right.$ $\left.r_{i}^{n}\right) R$. Since, for any $i=1, \ldots, k,\left(x^{n}-r_{i}^{n}\right) R+\bigcap_{j \neq i}\left(x^{n}-r_{j}^{n}\right) R=R$ we deduce that $\Phi$ is also onto. Therefore, by the first part of the proof, $\Phi$ induces an isomorphism $R / \operatorname{Ker} \Phi \cong$ $M_{n}\left(E^{\alpha}\right)^{k}$.

In Theorem 5.3 we will use the following examples.

Examples 3.3 Let $n, k \in \mathbb{N}$. 
(i) There exists a semilocal $\mathbb{Q}$-algebra $R$ that is a principal ideal domain such that $R / J(R) \cong$ $M_{n}(\mathbb{Q})^{k}$.

(ii) Let $F$ be any field, and consider the transcendental extension of $F, E=F\left(t_{1}, \ldots, t_{n}\right)$. Let $\alpha: E \rightarrow E$ be the automorphism of $E$ that fixes $F$ and satisfies that $\alpha\left(t_{i}\right)=t_{i+1}$ for $i=1, \ldots, n-1$ and $\alpha\left(t_{n}\right)=t_{1}$. Then there exists a semilocal $F$-algebra $R$, that is a principal ideal domain, such that $R / J(R) \cong M_{n}\left(E^{\alpha}\right)^{k}$.

In both cases all projective right or left modules over $R$ are free.

Proof. (i) Let $\mathbb{Q} \subseteq E$ be a Galois field extension with Galois group $G \cong \mathbb{Z} / n \mathbb{Z}$. Let $\alpha: E \rightarrow E$ be a generator of $G$. By Proposition 3.2, there exists an onto ring homomorphism $\varphi: E[x ; \alpha] \rightarrow M_{n}(\mathbb{Q})^{k}$. By Proposition $3.1 \Sigma=\left\{a \in R \mid \varphi(a)\right.$ is invertible in $\left.M_{n}(\mathbb{Q})^{k}\right\}$ is a right and left Ore set and $(E[x ; \alpha])_{\Sigma}$ has the desired properties.

(ii) Proceed as in (i) combining Proposition 3.2 with Proposition 3.1

\section{Pullbacks of rings}

We shall use ring pullbacks to construct noetherian semilocal rings with prescribed $V^{*}(R)$. In this section we study when ring pullbacks are semilocal and noetherian. We start fixing some notation.

Notation 4.1 Let $R_{1}, R_{2}$ and $S$ be rings with ring homomorphisms $j_{i}: R_{i} \rightarrow S$, for $i=1,2$. Let $R$ be the pullback of these rings. That is, $R$ fits into the pullback diagram

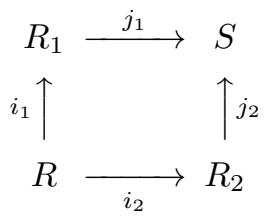

So that it can be described as

$$
R=\left\{\left(r_{1}, r_{2}\right) \in R_{1} \times R_{2} \mid j_{1}\left(r_{1}\right)=j_{2}\left(r_{2}\right)\right\}
$$

and the maps $i_{1}$ and $i_{2}$ are just the canonical projections.

A ring homomorphism $\varphi: R \rightarrow S$ is said to be local if, for any $r \in R, \varphi(r)$ is a unit of $S$ if and only if $r$ is a unit of $R$. The following deep result by Rosa Camps and Warren Dicks (see [5, Theorem 1] or [9, Theorem 4.2]) characterizes semilocal rings in terms of local morphisms.

Theorem 4.2 $A$ ring $R$ is semilocal if and only if it has a local ring homomorphism into a semilocal ring.

We note the following elegant corollary of Theorem 4.2 
Corollary 4.3 In the situation of Notation 4.1, $R$ is a local subring of $R_{1} \times R_{2}$. In particular, the pullback of two semilocal rings is a semilocal ring.

Proof. Note that if $\left(r_{1}, r_{2}\right) \in R \subseteq R_{1} \times R_{2}$ is a unit of $R_{1} \times R_{2}$ then its inverse $\left(r_{1}^{-1}, r_{2}^{-1}\right)$ also satisfies that $j_{1}\left(r_{1}^{-1}\right)=j_{2}\left(r_{2}^{-1}\right)$. Hence $\left(r_{1}^{-1}, r_{2}^{-1}\right) \in R$, and we deduce that the inclusion $R \rightarrow R_{1} \times R_{2}$ is a local ring homomorphism.

Lemma 4.4 Let $T$ be a subring of a ring $R$, and assume that there exists a two-sided ideal $I$ of $R$ such that $I \subseteq T$ and that $R / I$ is finitely generated as a left $T / I$-module. Then:

(i) ${ }_{T} R$ is finitely generated.

(ii) If $R$ and $T / I$ are left noetherian rings then so is $T$.

Proof. (i) Let $x_{1}, \ldots, x_{n} \in R$ be such that $x_{1}+I, \ldots, x_{n}+I$ generate $R / I$ as a left $T / I$-module. Then $R=T x_{1}+\cdots+T x_{n}+T \cdot 1$ is finitely generated as a left $T$-module.

(ii) Let $J$ be a left ideal of $T$. As $I J$ is a left ideal of $R$, it is finitely generated as a left $R$-module. By (i), it is finitely generated as a left $T$-module.

The left $R$-module $R J / I J$ is also a finitely generated left $R / I$-module, hence it is a noetherian left $T / I$-module. Therefore $J / I J \subseteq R J / I J$ is a finitely generated left $T / I$ module. Since

$$
0 \rightarrow I J \rightarrow J \rightarrow J / I J \rightarrow 0
$$

we can conclude that ${ }_{T} J$ is finitely generated. This proves that $T$ is left noetherian.

Proposition 4.5 In the situation of Notation 4.1. assume that $j_{1}$ is surjective and that ${ }_{R_{2}} S$ is finitely generated. If $R_{i}$ is a left noetherian ring, for $i=1,2$, then $R$ is left noetherian.

Proof. As $j_{1}$ is onto, $i_{2}$ is also an onto ring homomorphism with kernel Ker $j_{1} \times\{0\}$. Let $T=i_{1}(R)$, and note that $\operatorname{Ker} j_{1}$ is a two-sided ideal of $R_{1}$ that is contained in $T$. As a first step we shall prove that $T$ is left noetherian and that ${ }_{T} R_{1}$ is finitely generated. In view of Lemma 4.4 we only need to prove that ${ }_{T} S \cong R_{1} /$ Ker $j_{1}$ is finitely generated.

By assumption, there exist $s_{1}, \ldots, s_{n} \in S$ such that $S=\sum_{i=1}^{n} R_{2} s_{i}$. Fix an element $s \in S$, there exist $r_{2}^{1}, \ldots r_{2}^{n} \in R_{2}$ such that $s=\sum_{i=1}^{n} r_{2}^{i} \cdot s_{i}=\sum_{i=1}^{n} j_{2}\left(r_{2}^{i}\right) s_{i}$. Since $j_{1}$ is onto, for $i=1, \ldots, n$, there exists $r_{1}^{i} \in R_{1}$ such that $j_{1}\left(r_{1}^{i}\right)=j_{2}\left(r_{2}^{i}\right)$. Hence $s=\sum_{i=1}^{n} j_{1}\left(r_{1}^{i}\right) s_{i}=$ $\sum_{i=1}^{n} r_{1}^{i} \cdot s_{i}$. Since $\left(r_{1}^{i}, r_{2}^{i}\right) \in R, r_{1}^{i} \in T$ for $i=1, \ldots, n$. This shows that $S=\sum_{i=1}^{n} T s_{i}$, so that ${ }_{T} S$ is finitely generated.

We want to prove that any left ideal of $R$ is finitely generated. Let $I$ be a left ideal of $R$ contained in Ker $j_{1} \times\{0\}$. Hence $I=I_{1} \times 0$ with $I_{1}$ a left ideal of $T$, as $T$ is left noetherian $I$ is finitely generated.

Now, let $I$ be any left ideal of $R$. Since $i_{2}$ is onto and $R_{2}$ is left noetherian, $i_{2}(I)$ is a left ideal of $R_{2}$, finitely generated by elements $r_{2}^{1}, \ldots, r_{2}^{n} \in R_{2}$ say. Fix $r_{1}^{1}, \ldots, r_{1}^{n} \in R_{1}$ such that $\left(r_{1}^{i}, r_{2}^{i}\right) \in I$. If $x \in I$ then there exists $y_{1}, \ldots, y_{n} \in R$ such that $x-\sum_{i=1}^{n} y_{i}\left(r_{1}^{i}, r_{2}^{i}\right) \in$ 
$I \bigcap\left(\operatorname{Ker} j_{1} \times\{0\}\right)$. Therefore $I=I \bigcap\left(\operatorname{Ker} j_{1} \times\{0\}\right)+\sum_{i=1}^{n} R\left(r_{1}^{i}, r_{2}^{i}\right)$. By the previous case, $I \bigcap\left(\operatorname{Ker} j_{1} \times\{0\}\right)$ is finitely generated, therefore $I$ is finitely generated.

In the next result we compute the Jacobson radical for some pullbacks of rings.

Lemma 4.6 In the situation of Notation 4.1, assume that $j_{1}$ is an onto ring homomorphism such that $\operatorname{Ker} j_{1} \subseteq J\left(R_{1}\right)$ and $j_{1}\left(J\left(R_{1}\right)\right) \supseteq j_{2}\left(J\left(R_{2}\right)\right)$. Then $J(R)$ fits into the induced pullback diagram

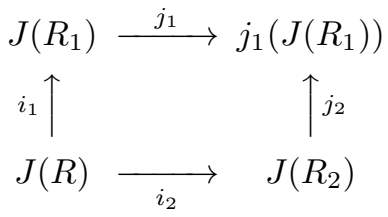

and $R / J(R) \cong R_{2} / J\left(R_{2}\right)$. In particular, if $J\left(R_{1}\right)=\operatorname{Ker} j_{1}$ and $J\left(R_{2}\right)=\operatorname{Ker} j_{2}$ then $J(R)=J\left(R_{1}\right) \times J\left(R_{2}\right)$.

Proof. Let $J$ be the pullback of the induced maps $j_{1}: J\left(R_{1}\right) \rightarrow j_{1}\left(J\left(R_{1}\right)\right)$ and $j_{2}: J\left(R_{2}\right) \rightarrow j_{1}\left(J\left(R_{1}\right)\right)$. Since, by Corollary 4.3, $R$ is a local subring of $R_{1} \times R_{2}$ it follows that $J \subseteq J(R)$.

In order to prove the reverse inclusion consider $\left(r_{1}, r_{2}\right) \in J(R)$. Being $j_{1}$ onto, $i_{2}$ is also onto, hence $r_{2} \in J\left(R_{2}\right)$. Since $j_{1}\left(r_{1}\right)=j_{2}\left(r_{2}\right) \subseteq j_{1}\left(J\left(R_{1}\right)\right)$, we deduce that $r_{1} \in$ $\operatorname{Ker} j_{1}+J\left(R_{1}\right)=J\left(R_{1}\right)$. Therefore $\left(r_{1}, r_{2}\right) \in J$.

Since Ker $i_{2}=\operatorname{Ker} j_{1} \times\{0\} \subseteq J(R)$ and $i_{2}$ is onto, it immediately follows that the map

$$
R \stackrel{i_{2}}{\rightarrow} R_{2} \stackrel{\pi}{\rightarrow} R_{2} / J\left(R_{2}\right),
$$

where $\pi$ denotes the canonical projection, induces an isomorphism between $R / J(R)$ and $R_{2} / J\left(R_{2}\right)$.

The claim when $J\left(R_{i}\right)=\operatorname{Ker} j_{i}$ follows from the fact that the pullback of zero homomorphisms is the product.

Milnor proved in 21] that, in the situation of Notation 4.1 and assuming that $j_{1}$ is onto, the category of projective modules over $R$ is the pullback of the category of projective modules over $R_{1}$ and of the category of projective modules over $R_{2}$. We are interested in the following version of this result,

Theorem 4.7 [21, Theorem 2.1] In the situation of Notation [4.1. assume that $j_{1}$ is an onto ring homomorphism. Then there are pullbacks of monoid morphisms



Remark 4.8 In fact, Milnor proved that having projective modules $P_{1}$ over $R_{1}$ and $P_{2}$ over $R_{2}$ such that $P_{1} \otimes_{R_{1}} S$ and $P_{2} \otimes_{R_{2}} S$ are isomorphic $S$-modules, then there exists a 
(unique) projective $R$-module $P$ such that $P_{1} \simeq P \otimes_{R} R_{1}$ and $P_{2} \simeq P \otimes_{R} R_{2}$. Moreover, if $P_{1}$ is finitely generated over $R_{1}$ and $P_{2}$ is finitely generated over $R_{2}$, then $P$ is finitely generated over $R$. He did not state explicitly that $P$ is countably generated $R$-module if $P_{1}$ is countably generated over $R_{1}$ and $P_{2}$ is countably generated $R_{2}$. However, it follows directly from his proof.

Now we state the precise result we will be using in $\$ 5$,

Corollary 4.9 In the situation of Notation 4.1, let $R_{1}$ and $R_{2}$ be semilocal rings, and let $S$ be a semisimple artinian ring. Fix an isomorphism $\varphi: S \rightarrow M_{m_{1}}\left(D_{1}^{\prime}\right) \times \cdots \times M_{m_{\ell}}\left(D_{\ell}^{\prime}\right)$ for suitable division rings $D_{1}^{\prime}, \ldots, D_{\ell}^{\prime}$. Assume that $j_{1}$ is an onto ring homomorphism with kernel $J\left(R_{1}\right)$, and that $J\left(R_{2}\right) \subseteq \operatorname{Ker} j_{2}$. If $R_{2} / J\left(R_{2}\right) \cong M_{n_{1}}\left(D_{1}\right) \times \cdots \times M_{n_{k}}\left(D_{k}\right)$ for $D_{1}, \ldots, D_{k}$ division rings, and $\pi: R_{2} \rightarrow M_{n_{1}}\left(D_{1}\right) \times \cdots \times M_{n_{k}}\left(D_{k}\right)$ is an onto morphism with kernel $J\left(R_{2}\right)$ then

(i) $i_{2}$ induces an onto ring homomorphism $\overline{i_{2}}: R \rightarrow M_{n_{1}}\left(D_{1}\right) \times \cdots \times M_{n_{k}}\left(D_{k}\right)$ with kernel $J(R)$. In particular, $R$ is a semilocal ring and $R / J(R) \cong R_{2} / J\left(R_{2}\right)$.

(ii) Let $\alpha: \operatorname{dim} \overline{i_{2}}: V^{*}\left(R_{2}\right) \rightarrow\left(\mathbb{N}_{0}^{*}\right)^{\ell}$ be the monoid homomorphism induced by $i_{2}$ and $\varphi$. Then

$$
\operatorname{dim} \bar{i}_{2} V^{*}(R)=\left\{x \in \operatorname{dim}_{\pi} V^{*}\left(R_{2}\right) \subseteq\left(\mathbb{N}_{0}^{*}\right)^{k} \mid \alpha(x) \in \operatorname{dim}_{\varphi j_{1}} V^{*}\left(R_{1}\right)\right\} .
$$

Moreover, if $R_{1}$ and $R_{2}$ are noetherian, and $S$ is finitely generated, both as a left and as a right $j_{2}\left(R_{2}\right)$-module, then $R$ is noetherian and $\operatorname{dim} \frac{-}{i_{2}}(V(R))=\operatorname{dim}-V_{i_{2}}^{*}(R) \cap\left(\mathbb{N}_{0}\right)^{k}$.

Proof. Statement $(i)$ follows from Lemma 4.6 and Corollary 4.3. Statement $(i i)$ is a consequence of Theorem 4.7 .

The final part of the Corollary follows from Proposition 4.5 and the fact that over a noetherian semilocal ring a projective module is finitely generated if and only if it is finitely generated modulo the Jacobson radical (cf. Proposition 1.9 or Theorem 2.3).

We single out the following particular case of Corollary 4.9,

Corollary 4.10 In the situation of Notation 4.1. for $i=1,2$, assume that $j_{i}$ is onto and Ker $j_{i}=J\left(R_{i}\right)$. Assume $S=M_{n_{1}}\left(D_{1}\right) \times \cdots \times M_{n_{k}}\left(D_{k}\right)$ where $D_{1}, \ldots, D_{k}$ are division rings. Then

(i) $i_{2}$ (and $i_{1}$ ) induces an onto ring homomorphism $\bar{i}: R \rightarrow M_{n_{1}}\left(D_{1}\right) \times \cdots \times M_{n_{k}}\left(D_{k}\right)$ with kernel $J(R)$.

(ii) $\operatorname{dim}_{\bar{i}} V^{*}(R)=\operatorname{dim}_{j_{1}} V^{*}\left(R_{1}\right) \cap \operatorname{dim}_{j_{2}} V^{*}\left(R_{2}\right)$.

\section{Noetherian semilocal rings with prescribed $V^{*}(R)$}

Now we have all the elements to construct noetherian semilocal rings with prescribed $V^{*}(R)$ and to prove the realization part of Theorem 2.7. We explain the basic constructions in the following two examples. 
Example 5.1 Let $k, m \in \mathbb{N}$, and let $a_{1}, \ldots, a_{k} \in \mathbb{N}_{0}$. Assume $\left(n_{1}, \ldots, n_{k}\right) \in \mathbb{N}^{k}$ is such that $a_{1} n_{1}+\cdots+a_{k} n_{k}=m \ell \in \mathbb{N}$. Let $F$ be a field, and let $F \subseteq F_{2}$ be a field extension such that there exist a semilocal principal ideal domain $R_{1}$, that is also an $F$-algebra, with $R_{1} / J\left(R_{1}\right) \cong M_{m}\left(F_{2}\right)$. Then for any intermediate field $F \subseteq F_{1} \subseteq F_{2}$ such that $\left[F_{2}: F_{1}\right]<\infty$ there exist a noetherian semilocal $F$-algebra $R$ and an onto ring homomorphism $\varphi: R \rightarrow$ $M_{n_{1}}\left(F_{1}\right) \times \cdots \times M_{n_{k}}\left(F_{1}\right)$ with $\operatorname{Ker} \varphi=J(R)$ such that $\operatorname{dim}_{\varphi} V^{*}(R)$ is exactly the set of solution in $\left(\mathbb{N}_{0}^{*}\right)^{k}$ of the congruence $a_{1} t_{1}+\cdots+a_{k} t_{k} \in m \mathbb{N}_{0}^{*}$.

Proof. Fix $j_{1}: M_{\ell}\left(R_{1}\right) \rightarrow M_{m \ell}\left(F_{2}\right)$ an onto ring homomorphism with kernel $J\left(M_{\ell}\left(R_{1}\right)\right)=$ $M_{\ell}\left(J\left(R_{1}\right)\right)$.

Set $R_{2}=M_{n_{1}}\left(F_{1}\right) \times \cdots \times M_{n_{k}}\left(F_{1}\right)$, and consider the morphism

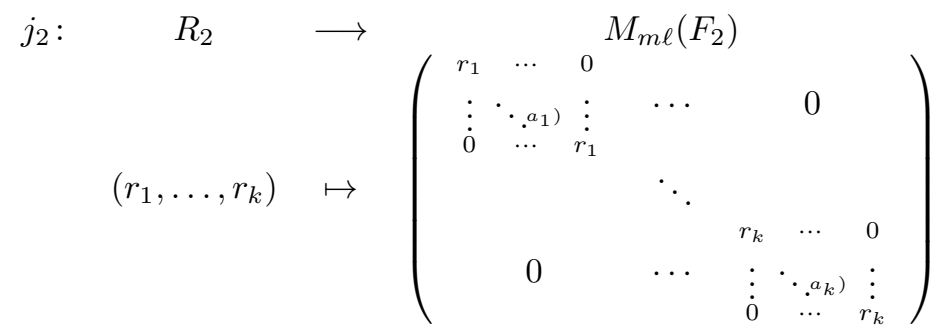

Note that $\left(V\left(R_{2}\right),\left\langle R_{2}\right\rangle\right) \cong\left(\mathbb{N}_{0}^{k},\left(n_{1}, \ldots, n_{k}\right)\right)$ and $V^{*}\left(R_{2}\right) \cong\left(\mathbb{N}_{0}^{*}\right)^{k} ; V\left(M_{m \ell}\left(F_{2}\right)\right)$ is isomorphic to the monoid $\mathbb{N}_{0}$ with order unit $m \cdot \ell$ and $V^{*}\left(M_{m \ell}\left(F_{2}\right)\right) \cong\left(\mathbb{N}_{0}\right)^{*}$. Then $j_{2}$ induces the morphism of monoids $f:\left(\mathbb{N}_{0}^{*}\right)^{k} \rightarrow \mathbb{N}_{0}^{*}$ defined by $f\left(x_{1}, \ldots, x_{k}\right)=a_{1} x_{1}+\cdots+a_{k} x_{k}$, cf. $\$ 1.1$.

Let $R$ be the ring defined by the pullback diagram

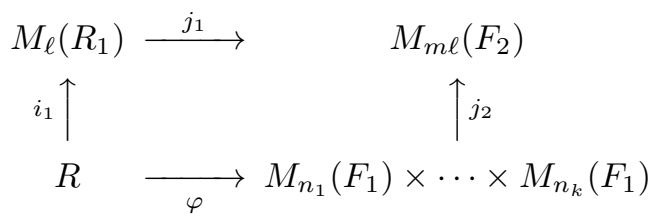

Since $\left[F_{2}: F_{1}\right]<\infty$ we can apply Corollary 4.9 to deduce $R$ is a noetherian semilocal $F$-algebra and that $\varphi$ is an onto ring homomorphism with kernel $J(R)$.

Now we compute $\operatorname{dim}_{\varphi} V^{*}(R)$ using also Corollary 4.9, We have chosen $R_{1}$ such that $\operatorname{dim}_{j_{1}} V^{*}\left(M_{\ell}\left(R_{1}\right)\right)=m \mathbb{N}_{0}^{*}$. Therefore $\left(x_{1}, \ldots, x_{k}\right) \in \operatorname{dim}_{\varphi} V^{*}(R)$ if and only if $f\left(x_{1}, \ldots, x_{k}\right)=$ $a_{1} x_{1}+\cdots+a_{k} x_{k} \in m \mathbb{N}_{0}^{*}$. That is, $\operatorname{dim}_{\varphi} V^{*}(R)$ is exactly the set of solutions in $\left(\mathbb{N}_{0}^{*}\right)^{k}$ of the congruence $a_{1} t_{1}+\cdots+a_{k} t_{k} \in m \mathbb{N}_{0}^{*}$ as desired.

Example 5.2 Let $k \in \mathbb{N}$, and let $a_{1}, \ldots, a_{k}, b_{1}, \ldots, b_{k} \in \mathbb{N}_{0}$. Let $\left(n_{1}, \ldots, n_{k}\right) \in \mathbb{N}^{k}$ be such that $a_{1} n_{1}+\cdots+a_{k} n_{k}=b_{1} n_{1}+\cdots+b_{k} n_{k} \in \mathbb{N}$. For any field extension $F \subseteq F_{1}$, there exist a noetherian semilocal $F$-algebra $R$ and an onto ring homomorphism $\varphi: R \rightarrow$ $M_{n_{1}}\left(F_{1}\right) \times \cdots \times M_{n_{k}}\left(F_{1}\right)$ with kernel $J(R)$ such that $\operatorname{dim}_{\varphi} V^{*}(R)$ is the set of solutions in $\left(\mathbb{N}_{0}^{*}\right)^{k}$ of the equation $a_{1} t_{1}+\cdots+a_{k} t_{k}=b_{1} t_{1}+\cdots+b_{k} t_{k}$.

Proof. Set $m=a_{1} n_{1}+\cdots+a_{k} n_{k}=b_{1} n_{1}+\cdots+b_{k} n_{k}$. 
Let $R_{1}$ be a noetherian semilocal $F$-algebra such that $R_{1} / J\left(R_{1}\right) \cong F_{1} \times F_{1}$, and all projective modules over $R_{1}$ are free. For example, we could take the commutative principal ideal domain $R_{1} \cong F_{1}[x]_{\Sigma}$ with $\Sigma=\left(F_{1}[x]\right) \backslash\left(x F_{1}[x] \cup(x-1) F_{1}[x]\right)$.

Let $j_{1}: M_{m}\left(R_{1}\right) \rightarrow M_{m}\left(F_{1}\right) \times M_{m}\left(F_{1}\right)$ be an onto ring homomorphism with kernel $J\left(M_{m}\left(R_{1}\right)\right)$. Set $R_{2}=M_{n_{1}}\left(F_{1}\right) \times \cdots \times M_{n_{k}}\left(F_{1}\right)$. Consider the morphism $j_{2}: R_{2} \longrightarrow$ $M_{m}\left(F_{1}\right) \times M_{m}\left(F_{1}\right)$ defined by

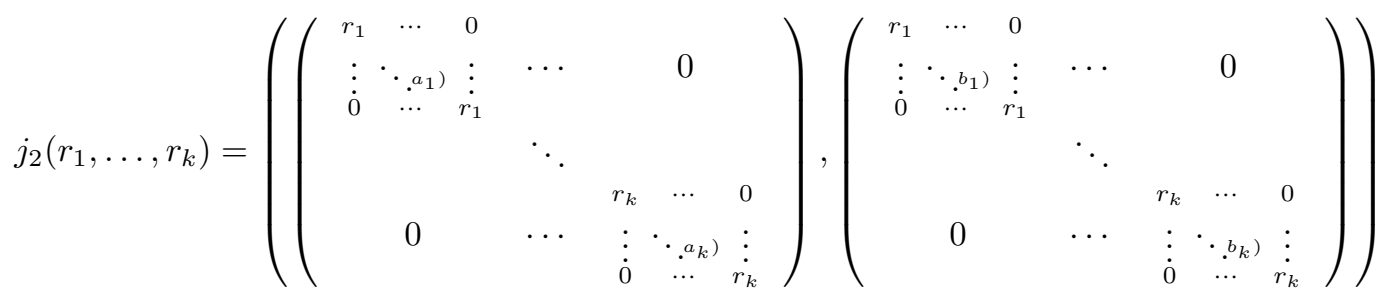

Note that $j_{2}$ induces the morphism of monoids $f:\left(\mathbb{N}_{0}^{*}\right)^{k} \rightarrow \mathbb{N}_{0}^{*} \times \mathbb{N}_{0}^{*}$ defined by $f\left(x_{1}, \ldots, x_{k}\right)=$ $\left(a_{1} x_{1}+\cdots+a_{k} x_{k}, b_{1} x_{1}+\cdots+b_{k} x_{k}\right)$, cf. 1.1 Hence, $f\left(n_{1}, \ldots, n_{k}\right)=(m, m)$.

Let $R$ be the ring defined by the pullback diagram

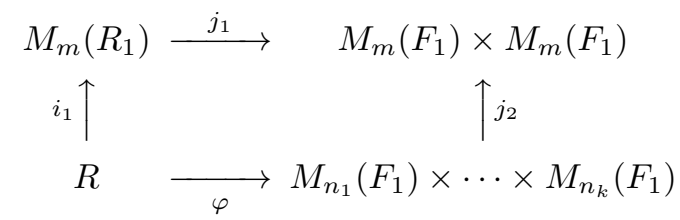

By Corollary 4.9, $R$ is a noetherian semilocal $F$-algebra and $\varphi$ is an onto ring homomorphism with kernel $J(R)$. We have chosen $R_{1}$ such that $\operatorname{dim}_{j_{1}} V^{*}\left(M_{\ell}\left(R_{1}\right)\right)=\{(x, x) \mid$ $\left.x \in \mathbb{N}_{0}^{*}\right\}$. Also by Corollary 4.9 $\left(x_{1}, \ldots, x_{k}\right) \in \operatorname{dim}_{\varphi} V^{*}(R)$ if and only if $f\left(x_{1}, \ldots, x_{k}\right) \in$ $\operatorname{dim}_{j_{1}} V^{*}\left(M_{\ell}\left(R_{1}\right)\right)$ if and only if $a_{1} x_{1}+\cdots+a_{k} x_{k}=b_{1} x_{1}+\cdots+b_{k} x_{k}$ as desired.

Theorem 5.3 Let $k \geq 1$, and let $F$ be a field. Let $M$ be a submonoid of $\left(\mathbb{N}_{0}^{*}\right)^{k}$ defined by a system of equations and containing an element $\left(n_{1}, \ldots, n_{k}\right) \in \mathbb{N}^{k}$. Then there exist a noetherian semilocal $F$-algebra $R$, a semisimple $F$-algebra $S=M_{n_{1}}(E) \times \cdots \times M_{n_{k}}(E)$, where $E$ is a field extension of $F$, and an onto morphism of $F$-algebras $\varphi: R \rightarrow S$ with $\operatorname{Ker} \varphi=J(R)$ satisfying that $\operatorname{dim}_{\varphi} V^{*}(R)=M$.

Proof. Let $M$ be defined by the system of equations,

$$
D\left(\begin{array}{c}
t_{1} \\
\vdots \\
t_{k}
\end{array}\right) \in\left(\begin{array}{c}
m_{1} \mathbb{N}_{0}^{*} \\
\vdots \\
m_{n} \mathbb{N}_{0}^{*}
\end{array}\right) \quad(*) \quad \text { and } \quad E_{1}\left(\begin{array}{c}
t_{1} \\
\vdots \\
t_{k}
\end{array}\right)=E_{2}\left(\begin{array}{c}
t_{1} \\
\vdots \\
t_{k}
\end{array}\right)
$$

where $D \in M_{n \times k}\left(\mathbb{N}_{0}\right), E_{1}, E_{2} \in M_{\ell \times k}\left(\mathbb{N}_{0}\right), n, \ell \geq 0$ and $m_{1}, \ldots, m_{n} \in \mathbb{N}, m_{i} \geq 2$ for any $i \in\{1, \ldots, n\}$.

Step 1. There exist a field $E$ containing $F$, a noetherian semilocal $F$-algebra $R_{1}$ and an onto ring homomorphism $\varphi_{1}: R_{1} \rightarrow M_{n_{1}}(E) \times \cdots \times M_{n_{k}}(E)$ such that $\operatorname{dim}_{\varphi_{1}} V^{*}\left(R_{1}\right)$ is the set of solution in $\left(\mathbb{N}_{0}^{*}\right)^{k}$ of the system of congruences $(*)$. 
If $n=0$, that is, if $(*)$ is empty we set $E=F, R_{1}=M_{n_{1}}(E) \times \cdots \times M_{n_{k}}(E)$ and $\varphi_{1}=$ Id. Assume $n>0$, therefore we may also assume that all the rows of $D$ are non zero.

Consider the field extension $F \subseteq F^{\prime}=F\left(t_{j}^{i} \mid i=1, \ldots, n, j=1, \ldots, m_{i}\right)$. For each $i=1, \ldots, n$ consider the automorphism $\alpha_{i}$ of $F^{\prime}$ that fixes $F_{i}=F\left(t_{j}^{s} \mid s \neq i\right) \subseteq F^{\prime}$, maps $t_{j}^{i}$ to $t_{j+1}^{i}$ for $1 \leq j<m_{i}$, and maps $t_{m_{i}}^{i}$ to $t_{1}^{i}$. Note that $\alpha_{i}$ has order $m_{i}$. Let $G$ be the group of permutations of $m_{1}+\cdots+m_{k}$ variables. Then $G$ acts on $F^{\prime}$. Set $E=\left(F^{\prime}\right)^{G}$, and note that $E \subseteq F^{\prime}$ is a finite field extension.

By Example 3.3(ii), for each $i=1, \ldots, n$, we can construct a principal ideal domain such that modulo the Jacobson radical is isomorphic to $M_{m_{i}}\left(\left(F^{\prime}\right)^{\alpha_{i}}\right)$. By Example [5.1] for $i=1, \ldots, n$, there exist a noetherian semilocal $F$-algebra $L_{i}$ and an onto ring morphism $\pi_{i}: L_{i} \rightarrow M_{n_{1}}(E) \times \cdots \times M_{n_{k}}(E)$ with kernel $J\left(L_{i}\right)$ and such that $\operatorname{dim}_{\pi_{i}} V^{*}\left(L_{i}\right)$ is the set of solutions in $\left(\mathbb{N}_{0}^{*}\right)^{k}$ of the $i$-th congruence in $(*)$.

Let $R_{1}$ be the pullback of $\pi_{i}, i=1, \ldots, n$. By Corollary 4.9, $R_{1}$ is a noetherian semilocal $F$-algebra. By Corollary 4.10, there exist an onto ring homomorphism $\varphi_{1}: R_{1} \rightarrow M_{n_{1}}(E) \times$ $\cdots \times M_{n_{k}}(E)$, with kernel $J\left(R_{1}\right)$, such that $\operatorname{dim}_{\varphi_{1}} V^{*}\left(R_{1}\right)$ is the monoid of solutions of $\left(^{*}\right)$. This concludes the proof of the first step.

Step 2. There exist a noetherian semilocal $F$-algebra $R_{2}$ and an onto ring homomorphism $\varphi_{2}: R_{2} \rightarrow M_{n_{1}}(E) \times \cdots \times M_{n_{k}}(E)$ such that $\operatorname{dim}_{\varphi_{2}} V^{*}\left(R_{2}\right)$ is the set of solution in $\left(\mathbb{N}_{0}^{*}\right)^{k}$ of the system of equations $(* *)$.

If $\ell=0$, that is, if $(* *)$ is empty we set $R_{2}=M_{n_{1}}(E) \times \cdots \times M_{n_{k}}(E)$ and $\varphi_{2}=$ Id. Assume $\ell>0$. Therefore, we can assume that none of the rows in $E_{1}$ and, hence, in $E_{2}$ are zero.

By Example 5.2, for $i=1, \ldots, \ell$, there exist a noetherian semilocal $F$-algebra $T_{i}$ and an onto ring morphism $\pi_{i}: T_{i} \rightarrow M_{n_{1}}(E) \times \cdots \times M_{n_{k}}(E)$ with kernel $J\left(T_{i}\right)$ and such that $\operatorname{dim}_{\pi_{i}} V^{*}\left(T_{i}\right)$ is the set of solutions in $\left(\mathbb{N}_{0}^{*}\right)^{k}$ of the $i$-th equation defined by the matrices $E_{1}$ and $E_{2}$.

Let $R_{2}$ be the pullback of $\pi_{i}, i=1, \ldots, \ell$. By Corollary $4.9, R_{2}$ is a noetherian semilocal $F$-algebra with an onto ring morphism $\varphi_{2}: R_{2} \rightarrow M_{n_{1}}(E) \times \cdots \times M_{n_{k}}(E)$ with kernel $J\left(R_{2}\right)$. By Corollary 4.10, $\operatorname{dim}_{\varphi_{2}} V^{*}\left(R_{2}\right)$ is the set of solutions of $(* *)$. This concludes the proof of step 2.

Finally, set $R$ to be the pullback of $\varphi_{i}: R_{i} \rightarrow M_{n_{1}}(E) \times \cdots \times M_{n_{k}}(E), i=1,2$. By Corollary 4.9, $R$ is a noetherian semilocal $F$-algebra with an onto ring morphism $\varphi: R \rightarrow$ $M_{n_{1}}(E) \times \cdots \times M_{n_{k}}(E)$ with kernel $J(R)$. By Corollary 4.10, the elements in $\operatorname{dim}_{\varphi} V^{*}(R)$ are the solutions of $(*)$ and $(* *)$.

\section{Solving systems in $\mathbb{N}_{0}$ and in $\mathbb{N}_{0}^{*}$ : Supports of solutions}

In this section we study the supports of the elements in monoids defined by systems of equations. Our main aim is to show in Proposition 6.7 that if $A$ is a full affine submonoid of $\mathbb{N}_{0}^{k}$ then $A+\infty A$ is a monoid defined by a system of equations. 
Next result is quite easy but it is very important to keep it in mind, for example in Definition 7.1. It shows that full affine submonoids are closed by projections over the complementary of supports of elements.

Proposition 6.1 Let $k \geq 1$. Let $A \subseteq \mathbb{N}_{0}^{k}$ be a full affine submonoid. Let $I \varsubsetneqq\{1, \ldots, k\}$ be the support of an element of $A$, and denote by $p_{I}: \mathbb{N}_{0}^{k} \rightarrow \mathbb{N}_{0}^{\{1, \ldots, k\} \backslash I}$ the canonical projection. Then $p_{I}(A)$ is a full affine submonoid of $\mathbb{N}_{0}^{\{1, \ldots, k\} \backslash I}$.

Proof. Clearly $p_{I}(A)$ is a submonoid of $\mathbb{N}_{0}^{\{1, \ldots, k\} \backslash I}$. We need to check the full affine property.

Let $a, b \in A$ be such that there exists $z \in \mathbb{N}_{0}^{\{1, \ldots, k\} \backslash I}$ satisfying that $p_{I}(a)+z=p_{I}(b)$. Let $d \in A$ be such that $\operatorname{supp}(d)=I$. There exists $n \in \mathbb{N}_{0}$ such that $a+c=b+n d$ for some $c \in \mathbb{N}_{0}^{k}$. As $A$ is full affine, $c \in A$ and, hence, $z=p_{I}(c) \in p_{I}(A)$.

Let $C \subseteq \mathbb{Q}^{k}$, and let $C^{\perp}=\left\{v \in \mathbb{Q}^{k} \mid\langle v, c\rangle=0\right.$ for any $\left.c \in C\right\}$ where $\langle-,-\rangle$ denotes the standard scalar product. If $X \subseteq \mathbb{Q}^{k}$, the support of $X$ is defined by

$$
\operatorname{supp}(X)=\bigcup_{x \in X} \operatorname{supp}(x)
$$

Let $A$ be a submonoid of $\mathbb{N}_{0}^{k}$, and let $B$ be the subgroup of $\mathbb{Z}^{k}$ generated by $A$. Then $A$ is full affine if and only if $B \cap \mathbb{N}_{0}^{k}=A$ (see, for example, [10, Lemma 3.1]). So assume $A$ is full affine and consider $B^{\prime}=\left(A^{\perp}\right)^{\perp}$ which is a subgroup of $\mathbb{Z}^{k}$, containing $B$, defined by a system of diophantine linear equations

$$
E\left(\begin{array}{c}
t_{1} \\
\vdots \\
t_{k}
\end{array}\right)=\left(\begin{array}{c}
0 \\
\vdots \\
0
\end{array}\right)
$$

with $E \in M_{(k-\ell) \times k}(\mathbb{Z})$ and $\ell$ is the rank of the group $B^{\prime}$.

Since the rank of $B$ is also $\ell$, there exist $d_{1}, \ldots, d_{\ell} \geq 1$ such that $B^{\prime} / B \cong \mathbb{Z} / d_{1} \mathbb{Z} \times \cdots \times$ $\mathbb{Z} / d_{\ell} \mathbb{Z}$. Equivalently, there exists a basis $v_{1}, \ldots, v_{\ell}$ of $B^{\prime}$ such that $d_{1} v_{1}, \ldots, d_{\ell} v_{\ell}$ is a basis of $B$. Therefore, an element $x \in B^{\prime}$

$$
x=\left(x_{1}, \ldots, x_{k}\right)=\alpha_{1} v_{1}+\cdots+\alpha_{\ell} v_{\ell}
$$

is in $B$ if and only if, for any $i=1, \ldots, \ell, \alpha_{i} \in d_{i} \mathbb{Z}$. Since each $\alpha_{i}$ can be written as a $\mathbb{Q}$-linear combination of $x_{1}, \ldots, x_{k}$, by clearing denominators and eliminating trivial congruences, we deduce that, for any $x \in B^{\prime}, x \in B$ if and only if it is a solution of

$$
D\left(\begin{array}{c}
t_{1} \\
\vdots \\
t_{k}
\end{array}\right) \in\left(\begin{array}{c}
m_{1} \mathbb{Z} \\
\vdots \\
m_{n} \mathbb{Z}
\end{array}\right)
$$

where $0 \leq n \leq \ell, D \in M_{n \times k}(\mathbb{Z})$ and $m_{i}>1$ for $i=1, \ldots, n$.

Adding to $D$ a suitable integral matrix of the form $\left(\begin{array}{ccc}m_{1} & \cdots & 0 \\ \vdots & \ddots & \vdots \\ 0 & \cdots & m_{n}\end{array}\right) \cdot A$, we can also assume that $D \in M_{n \times k}\left(\mathbb{N}_{0}\right)$.

In the next Proposition we collect the consequences of this discussion. 
Proposition 6.2 ([3, Exercise 6.4.16] or [2, Proof of Theorem 2.29]) Let $k \geq 1$. Let A be a full affine submonoid of $\mathbb{N}_{0}^{k}$, and let $\ell$ be the rank of the group generated by $A$. Then there exist $0 \leq n \leq \ell, D \in M_{n \times k}\left(\mathbb{N}_{0}\right), E_{1}, E_{2} \in M_{(k-\ell) \times k}\left(\mathbb{N}_{0}\right)$ and $m_{1}, \ldots, m_{n}$ integers strictly bigger than one such that

(i) $x=\left(t_{1}, \ldots, t_{k}\right) \in \mathbb{N}_{0}^{k}$ is an element of $A$ if and only if it is a solution of

$$
D \cdot T \in\left(\begin{array}{c}
m_{1} \mathbb{N}_{0} \\
\vdots \\
m_{n} \mathbb{N}_{0}
\end{array}\right) \quad \text { and } \quad E_{1} \cdot T=E_{2} \cdot T
$$

where $T=\left(t_{1}, \ldots, t_{k}\right)^{t}$.

For $j=1,2$, let $r_{i}^{j}$ denote the $i$-th row of $E_{j}$. Then $E_{1}$ and $E_{2}$ can be chosen such that, for $i=1, \ldots, k-\ell, \operatorname{supp}\left(r_{i}^{1}\right) \cap \operatorname{supp}\left(r_{i}^{2}\right)=\emptyset$.

(ii) The set of solutions of $E_{1} \cdot T=E_{2} \cdot T$ is $A^{\prime}=\left(A^{\perp}\right)^{\perp} \cap \mathbb{N}_{0}^{k}$.

(iii) There exists $d \in \mathbb{N}$ such that $d A^{\prime} \subseteq A$. In particular,

$$
\begin{aligned}
& \{I \subseteq\{1, \ldots, k\} \mid \text { there exists } a \in A \text { such that } \operatorname{supp}(a)=I\}= \\
& =\left\{I \subseteq\{1, \ldots, k\} \mid \text { there exists } a \in A^{\prime} \text { such that } \operatorname{supp}(a)=I\right\} .
\end{aligned}
$$

Proof. Following with the notation in the remarks before Proposition 6.2 we can write the matrix $E=E_{1}-E_{2}$ where $E_{1}$ and $E_{2}$ are in $M_{(k-\ell) \times k}\left(\mathbb{N}_{0}\right)$. Clearly, $E_{1}$ and $E_{2}$ can be chosen in a way such that the $i$-th row of $E_{1}$ has disjoint support with the $i$-th row of $E_{2}$. Then ( $i$ ) follows from the fact that $A=B \cap \mathbb{N}_{0}^{k}$.

The rest of the statement is clear.

Now we prove an auxiliary (and probably known) result that will be useful to determine the supports of positive solutions of linear diophantine equations.

Lemma 6.3 Let $k \geq 1$, and let $V$ be a subspace of $\mathbb{Q}^{k}$. Then the following statements are equivalent,

(i) $V^{\perp} \cap \mathbb{N}^{k} \neq \emptyset$;

(ii) $\operatorname{supp}\left(V^{\perp} \cap \mathbb{N}_{0}^{k}\right)=\{1, \ldots, k\}$;

(iii) $V \cap \mathbb{N}_{0}^{k}=\{0\}$.

Proof. It is clear that (i) and (ii) are equivalent statements and also that (i) implies (iii). We will show that (iii) implies (ii).

The assumption in (iii) is equivalent to say that any element $0 \neq v \in V$ has a component strictly bigger that zero an another one strictly smaller that zero. As a first step we show that $V^{\perp}$ cannot satisfy this condition. 
Assume, by the way of contradiction, that $k$ is the minimal dimension in which the conclusion of (ii) fails. So that there exists $V \leq \mathbb{Q}^{k}$ such that $V \cap \mathbb{N}_{0}^{k}=V^{\perp} \cap \mathbb{N}_{0}^{k}=\{0\}$. Note that $k$ and the dimension of $V$ must be strictly bigger than 1 .

Let $v_{1}, \ldots, v_{n}$ be a basis of $V$ such that there exists $i \in \operatorname{supp}\left(v_{1}\right) \backslash \operatorname{supp}\left(\left\{v_{2}, \ldots, v_{n}\right\}\right)$. Let $\pi: \mathbb{Q}^{k} \rightarrow \mathbb{Q}^{\{1, \ldots, k\} \backslash\{i\}}$ denote the canonical projection.

If $\pi(V) \cap \mathbb{N}_{0}^{\{1, \ldots, k\} \backslash\{i\}}=\{0\}$ then, by the minimality of $k$, there exists $v \in \mathbb{Q}^{k}$ such that $0 \neq \pi(v) \in \pi(V)^{\perp} \cap \mathbb{N}_{0}^{\{1, \ldots, k\} \backslash\{i\}}$. Since $v$ can be chosen satisfying that $i \notin \operatorname{supp}(v)$, we would get $0 \neq v \in V^{\perp} \cap \mathbb{N}_{0}^{k}$, a contradiction. Let $0 \neq \lambda_{1} \pi\left(v_{1}\right)+\cdots+\lambda_{n} \pi\left(v_{n}\right) \in \pi(V) \cap \mathbb{N}_{0}^{\{1, \ldots, k\} \backslash\{i\}}$. Then $w=\lambda_{1} v_{1}+\cdots+\lambda_{n} v_{n} \in V$. Since $V \cap \mathbb{N}_{0}^{k}=\{0\}, \lambda_{1} \neq 0$. Therefore, replacing $v_{1}$ by $w$ if necessary, we may assume that $0 \neq \pi\left(v_{1}\right) \in \mathbb{N}_{0}^{\{1, \ldots, k\} \backslash\{i\}}$ and that the $i$-th component of $v_{1}$ is $<0$. Let $-a$ be such component.

Let $W$ be the subspace of $\mathbb{Q}^{k \backslash\{i\}}$ generated by $\pi\left(v_{2}\right), \ldots, \pi\left(v_{n}\right)$. Our hypothesis imply that $W \cap \mathbb{N}_{0}^{\{1, \ldots, k\} \backslash\{i\}}=\{0\}$. By the minimality of $k$, there is $v \in \mathbb{Q}^{k}$ such that $0 \neq$ $\pi(v) \in W^{\perp} \cap \mathbb{N}_{0}^{\{1, \ldots, k\} \backslash\{i\}}$. Therefore, as $b=\left\langle\pi(v), \pi\left(v_{1}\right)\right\rangle \geq 0$, picking $v$ such that its $i$-th component is $b / a$ we find that $0 \neq a v \in V^{\perp} \cap \mathbb{N}_{0}^{k}$, a contradiction. Therefore $V^{\perp} \cap \mathbb{N}_{0}^{k} \neq\{0\}$ for any $V$ such that $V \cap \mathbb{N}_{0}^{k}=\{0\}$, as claimed.

Now assume that $V$ is a $\mathbb{Q}$-vector space satisfying (iii). Observe first that $\operatorname{supp}\left(V^{\perp}\right)=$ $\{1, \ldots, k\}$, since otherwise there would exist $i \in\{1, \ldots, k\}$ such that the $i$-th component of any element in $V^{\perp}$ is zero. Therefore, $e_{i}=\left(0, \ldots, 0,1^{i)}, 0, \ldots, 0\right) \in\left(V^{\perp}\right)^{\perp}=V$, which is a contradiction with the assumption.

Let $I=\operatorname{supp}\left(V^{\perp} \cap \mathbb{N}_{0}^{k}\right)$ and let $J=\{1, \ldots, k\} \backslash I$. We already know that $I \neq \emptyset$ and we want to show $J=\emptyset$. Assume, by the way of contradiction, that $J \neq \emptyset$. Set $\pi_{I}: \mathbb{Q}^{k} \rightarrow \mathbb{Q}^{I}$ and $\pi_{J}: \mathbb{Q}^{k} \rightarrow \mathbb{Q}^{J}$ to be the canonical projections.

Pick $x \in V^{\perp} \cap \mathbb{N}_{0}^{k}$ such that $\operatorname{supp}(x)=I$. Then for any $v \in V^{\perp}$ there exists $n \in \mathbb{N}_{0}$ such that $\pi_{I}(n x+v) \geq 0$. By the definition of $I$, this implies that $\pi_{J}(v)=\pi_{J}(n x+v)$ is either zero or it has a component $>0$ and another one $<0$. Therefore, $\pi_{J}\left(V^{\perp}\right) \cap \mathbb{N}_{0}^{J}=\{0\}$. By the first part of the proof, there exists $w \in \mathbb{Q}^{k}$ such that $0 \neq \pi_{J}(w) \in \pi_{J}\left(V^{\perp}\right)^{\perp} \cap \mathbb{N}_{0}^{J}$. Choosing $w$ such that $\pi_{I}(w)=0$, we obtain that $0 \neq w \in\left(V^{\perp}\right)^{\perp} \cap \mathbb{N}_{0}^{k}=V \cap \mathbb{N}_{0}^{k}$ which contradicts (iii). Therefore $J=\emptyset$.

Lemma 6.3 yields a first characterization of the supports of the elements in a full affine monoid. In following statements if $X \subseteq\left(\mathbb{N}_{0}^{*}\right)^{k}$, Supp $(X) \subseteq 2^{\{1, \ldots, k\}}$ is the set of supports of elements in $X$.

Corollary 6.4 Let $k \geq 1$. Let $A$ be a full affine submonoid of $\mathbb{N}_{0}^{k}$. Let $\emptyset \neq I \subseteq\{1, \ldots, k\}$, and denote by $\pi_{I}: \mathbb{Q}^{k} \rightarrow \mathbb{Q}^{I}$ the canonical projection. Then $I \in \operatorname{Supp}(A)$ if and only if $\pi_{I}(A)^{\perp} \cap \mathbb{N}_{0}^{I}=\{0\}$.

Proof. If $a \in A$ is such that $\operatorname{supp}(a)=I$ then, as $\left\langle x, \pi_{I}(a)\right\rangle=0$ for any $x \in \pi_{I}(A)^{\perp}$, it follows that $\pi_{I}(A)^{\perp} \cap \mathbb{N}_{0}^{I}=\{0\}$.

Conversely, if $\pi_{I}(A)^{\perp} \cap \mathbb{N}_{0}^{I}=\{0\}$ then, by Lemma 6.3, there exists $u \in\left(\pi_{I}(A)^{\perp}\right)^{\perp} \cap \mathbb{N}^{I}$. Let $x=\left(x_{1}, \ldots, x_{k}\right) \in \mathbb{N}_{0}^{k}$ be such that $\pi_{I}(x)=u$ and $x_{i}=0$ for any $i \in\{1, \ldots, k\} \backslash I$. Then $x \in\left(A^{\perp}\right)^{\perp} \cap \mathbb{N}_{0}^{k}$. By Proposition 6.2 (iii), there exists $d \in \mathbb{N}$ such that $a=d x \in A$. By construction, $\operatorname{supp}(a)=I$. 
A further characterization is the following.

Corollary 6.5 Let $k \geq 1$. Let $A$ be a full affine submonoid of $\mathbb{N}_{0}^{k}$. For any $\emptyset \neq I \subseteq$ $\{1, \ldots, k\}$ denote by $\pi_{I}: \mathbb{Q}^{k} \rightarrow \mathbb{Q}^{I}$ the canonical projection. Let $v_{1}, \ldots, v_{r} \in \mathbb{Q}^{k}$ be a finite subset of $A^{\perp}$. Then, there exist $v_{r+1}, \ldots, v_{s} \in A^{\perp}$ such that $v_{1}, \ldots v_{s}$ generate the $\mathbb{Q}$-vector space $A^{\perp}$ and the set

$$
\begin{gathered}
\mathcal{S}\left(v_{1}, \ldots, v_{s}\right)=\left\{\emptyset \neq I \subseteq\{1, \ldots, k\} \mid \text { for any } i=1, \ldots, s, \pi_{I}\left(v_{i}\right)\right. \text { is either zero } \\
\text { or it has a component }<0 \text { and a component }>0\}
\end{gathered}
$$

coincides with $\operatorname{Supp}(A \backslash\{0\})$.

Proof. Pick $v_{r+1}, \ldots, v_{s} \in A^{\perp}$ such that $v_{1}, \ldots, v_{s}$ generate $A^{\perp}$ and the set $\mathcal{S}\left(v_{1}, \ldots, v_{s}\right)$ has minimal cardinality.

We claim that $v_{1}, \ldots, v_{s}$ have the desired property. If $\emptyset \neq I \subseteq\{1, \ldots, k\}$ is such that there exist $a \in A$ such that $\operatorname{supp}(a)=I$ then $I \in \mathcal{S}\left(v_{1}, \ldots, v_{s}\right)$ because $\left\langle a, v_{i}\right\rangle=0$ for any $i=1, \ldots, s$.

Let $I \in \mathcal{S}\left(v_{1}, \ldots, v_{s}\right)$ and assume, by the way of contradiction, that $I \notin \operatorname{Supp}(A)$. Then $\pi_{I}\left(A^{\perp}\right) \cap \mathbb{N}_{0}^{I} \neq\{0\}$, because otherwise

$$
\pi_{I}(A)^{\perp} \cap \mathbb{N}_{0}^{I} \subseteq \pi_{I}\left(A^{\perp}\right) \cap \mathbb{N}_{0}^{I}=\{0\}
$$

and, by Corollary 6.4, this implies that there exists $a \in A$ with supp $(a)=I$.

Let $v \in A^{\perp}$ be such that $0 \neq \pi_{I}(v) \in \pi_{I}\left(A^{\perp}\right) \cap \mathbb{N}_{0}^{I}$. Notice that, $\mathcal{S}\left(v_{1}, \ldots, v_{s}, v\right) \subseteq$ $\mathcal{S}\left(v_{1}, \ldots, v_{s}\right)$ and $I \in \mathcal{S}\left(v_{1}, \ldots, v_{s}\right) \backslash \mathcal{S}\left(v_{1}, \ldots, v_{s}, v\right)$. This contradicts the minimality of the cardinality of $\mathcal{S}\left(v_{1}, \ldots, v_{s}\right)$. This finishes the proof of the claim and of the Corollary.

Now we move to consider the solutions over $\left(\mathbb{N}_{0}^{*}\right)^{k}$. In the next lemma we see how to determine the set of nonempty supports of such monoids, which coincide with the set of infinite supports, for the special kind of systems that appear in Proposition 6.2(i).

Lemma 6.6 Let $k \geq 1$. Let $M$ be a submonoid of $\left(\mathbb{N}_{0}^{*}\right)^{k}$ defined by a system of equations

$$
D \cdot T \in\left(\begin{array}{c}
m_{1} \mathbb{N}_{0}^{*} \\
\vdots \\
m_{n} \mathbb{N}_{0}^{*}
\end{array}\right) \quad(*) \quad \text { and } \quad E_{1} \cdot T=E_{2} \cdot T \quad(* *)
$$

where $D \in M_{n \times k}\left(\mathbb{N}_{0}\right), E_{1}, E_{2} \in M_{\ell \times k}\left(\mathbb{N}_{0}\right)$ and $m_{1}, \ldots, m_{n} \in \mathbb{N}, m_{i} \geq 2$ for any $i \in$ $\{1, \ldots, n\}$. For $j=1,2$ and $i=1, \ldots, \ell$, let $r_{i}^{j}$ denote the $i$-th row of $E_{j}$. For $\emptyset \neq I \subseteq$ $\{1, \ldots, k\}$, let $\pi_{I}:\left(\mathbb{N}_{0}^{*}\right)^{k} \rightarrow\left(\mathbb{N}_{0}^{*}\right)^{I}$ denote the canonical projection. Then:

(i) Let $N$ be the submonoid of $\left(\mathbb{N}_{0}^{*}\right)^{k}$ whose elements are the solutions of the system of congruences $(*)$. Then, for any $i \in\{1, \ldots, k\}$, the element $(0, \ldots, 0, \infty, 0, \ldots, 0) \in N$.

(ii) If $x \in M$ then also $\infty \cdot x \in M$. 
(iii) If $x \in M$ then the element $x^{*} \in\left(\mathbb{N}_{0}^{*}\right)^{k}$ uniquely determined by the property $\operatorname{supp} x^{*}=$ $\inf -\operatorname{supp} x^{*}=\inf -\operatorname{supp} x$ also belongs to $M$.

(iv) Assume that, for $i=1, \ldots, \ell, \operatorname{supp}\left(r_{i}^{1}\right) \cap \operatorname{supp}\left(r_{i}^{2}\right)=\emptyset$, and let $\emptyset \neq I \subseteq\{1, \ldots, k\}$ then there exists $x \in M$ such that $\operatorname{supp}(x)=I$ if and only if, for any $i=1, \ldots, \ell$, $\pi_{I}\left(r_{i}^{1}-r_{2}^{2}\right)$ is either 0 or it has a component $>0$ and another one $<0$.

Proof. Statement (i) is trivial, and it allows us to prove the rest of the statement just for $M$ defined by the system of linear diophantine equations $(* *)$.

Let $x \in M$. Fix $i \in\{1, \ldots, \ell\}$, there are three possible situations. The first one is $0=\left\langle x, r_{i}^{1}\right\rangle=\left\langle x, r_{i}^{2}\right\rangle$ which happens if and only if, for $j=1,2, \operatorname{supp}(x) \cap \operatorname{supp}\left(r_{i}^{j}\right)=\emptyset$. The second one is $0 \neq\left\langle x, r_{i}^{1}\right\rangle=\left\langle x, r_{i}^{2}\right\rangle \in \mathbb{N}_{0}$ which happens if and only if, for $j=1,2$, $\inf -\operatorname{supp}(x) \cap \operatorname{supp}\left(r_{i}^{j}\right)=\emptyset$ but $\operatorname{supp}(x) \cap \operatorname{supp}\left(r_{i}^{j}\right) \neq \emptyset$. Finally, $\left\langle x, r_{i}^{1}\right\rangle=\left\langle x, r_{i}^{2}\right\rangle=\infty$ if and only if $\inf -\operatorname{supp}(x) \cap \operatorname{supp}\left(r_{i}^{j}\right) \neq \emptyset$ for $j=1,2$. Then, in the three situations, it also follows that $\left\langle\infty \cdot x, r_{i}^{1}\right\rangle=\left\langle\infty \cdot x, r_{i}^{2}\right\rangle$ and $\left\langle x^{*}, r_{i}^{1}\right\rangle=\left\langle x^{*}, r_{i}^{2}\right\rangle$. This shows that (ii) and (iii) hold.

To prove statement (iv) assume that $\operatorname{supp}\left(r_{i}^{1}\right) \cap \operatorname{supp}\left(r_{i}^{2}\right)=\emptyset$ for $i=1, \ldots, \ell$. Let $\emptyset \neq I \subseteq\{1, \ldots, k\}$ have the property required in the statement. Let $x \in\left(\mathbb{N}_{0}^{*}\right)^{k}$ be such that $\operatorname{supp}(x)=\inf -\operatorname{supp}(x)=I$. If $i \in\{1, \ldots, \ell\}$ is such that $\pi_{I}\left(r_{i}^{1}-r_{i}^{2}\right)$ is zero then $0=\left\langle x, r_{i}^{1}\right\rangle=\left\langle x, r_{i}^{2}\right\rangle$, if $\pi_{I}\left(r_{i}^{1}-r_{i}^{2}\right)$ has a positive component and a negative component then $\infty=\left\langle x, r_{i}^{1}\right\rangle=\left\langle x, r_{i}^{2}\right\rangle$. This shows that $x$ satisfies $(* *)$, therefore it is an element of $M$.

To prove the converse, let $x \in M$. By (ii) we may assume that $x=\infty \cdot x$. Let $I=\operatorname{supp}(x)$, then one can proceed as in the proof of $(i i)$ and (iii) to show that $I$ has the required property.

Proposition 6.7 Let $k \geq 1$, and let $A$ be a full affine submonoid of $\mathbb{N}_{0}^{k}$. Then

$$
M=A+\{\infty \cdot a \mid a \in A\}
$$

is a submonoid of $\left(\mathbb{N}_{0}^{*}\right)^{k}$ defined by a system of equations.

Proof. We divide the proof into a couple of steps.

Step 1. Let $k \geq 1$, and let $A$ be a full affine submonoid of $\mathbb{N}_{0}^{k}$. Then there exists a submonoid $M^{\prime}$ of $\left(\mathbb{N}_{0}^{*}\right)^{k}$ defined by a system of equations such that $M^{\prime} \cap \mathbb{N}_{0}^{k}=A$ and if $x \in M^{\prime}$ then there exists $a \in A$ such that $\infty \cdot x=\infty \cdot a \in M^{\prime}$.

By Proposition 6.2 there exist $D \in M_{n \times k}\left(\mathbb{N}_{0}\right), E_{1}, E_{2} \in M_{\ell \times k}\left(\mathbb{N}_{0}\right)$ and $m_{1}, \ldots, m_{n} \in \mathbb{N}$, $m_{i} \geq 2$ for any $i \in\{1, \ldots, n\}$ such that the elements of $A$ are the solutions of the system

$$
D\left(\begin{array}{c}
t_{1} \\
\vdots \\
t_{k}
\end{array}\right) \in\left(\begin{array}{c}
m_{1} \mathbb{N}_{0}^{*} \\
\vdots \\
m_{n} \mathbb{N}_{0}^{*}
\end{array}\right) \quad(*) \quad \text { and } \quad E_{1}\left(\begin{array}{c}
t_{1} \\
\vdots \\
t_{k}
\end{array}\right)=E_{2}\left(\begin{array}{c}
t_{1} \\
\vdots \\
t_{k}
\end{array}\right)
$$

For $j=1,2$, let $r_{i}^{j}$ denote the $i$-th row of $E_{j}$. By Proposition 6.2 we can assume that, for $i=1, \ldots, \ell, \operatorname{supp}\left(r_{i}^{1}\right) \cap \operatorname{supp}\left(r_{i}^{1}\right)=\emptyset$. Set $v_{1}=r_{1}^{1}-r_{1}^{2}, \ldots, v_{\ell}=r_{\ell}^{1}-r_{\ell}^{2}$. Notice that $v_{1}, \ldots, v_{\ell} \in A^{\perp}$ and, in fact, generate $A^{\perp}$. 
By Corollary 6.5 there exist $v_{\ell+1}, \ldots, v_{s} \in A^{\perp}$ such that the set of supports of nonzero elements in $A$ coincides with

$$
\begin{aligned}
\mathcal{S}\left(v_{1}, \ldots, v_{s}\right) & =\left\{\emptyset \neq I \subseteq\{1, \ldots, k\} \mid \text { for any } i=1, \ldots, s, \pi_{I}\left(v_{i}\right)\right. \text { is either zero } \\
& \text { or it has a component }<0 \text { and a component }>0\}
\end{aligned}
$$

Now, for $i=\ell+1, \ldots, s$, write $v_{i}=r_{i}^{1}-r_{i}^{2}$ where $r_{i}^{j} \in \mathbb{N}_{0}^{k}$ and $\operatorname{supp}\left(r_{i}^{1}\right) \cap \operatorname{supp}\left(r_{i}^{1}\right)=\emptyset$. For, $j=1,2$, let $F_{j}$ be the matrix whose $i$-th row is $r_{\ell+i}^{j}$. Now, add to the initial system defining $A$ the equations defined by

$$
F_{1}\left(\begin{array}{c}
t_{1} \\
\vdots \\
t_{k}
\end{array}\right)=F_{2}\left(\begin{array}{c}
t_{1} \\
\vdots \\
t_{k}
\end{array}\right)
$$

Let $M^{\prime}$ be the set of solutions in $\left(\mathbb{N}_{0}^{*}\right)^{k}$, and note that $A$ is still the set of solutions in $\mathbb{N}_{0}^{k}$ of this system so that $M^{\prime} \cap \mathbb{N}_{0}^{k}=A$. By Lemma 6.6(iv), the set of supports of elements of $M^{\prime}$ is exactly $\mathcal{S}\left(v_{1}, \ldots, v_{s}\right)$ which, by construction, coincides with the set of supports of elements in $A$. This implies that if $x \in M^{\prime}$ then there exists $a \in A$ such that $\infty \cdot x=\infty \cdot a$. By Lemma 6.6, if $x \in M^{\prime}$ then $\infty \cdot x \in M^{\prime}$. This finishes the proof of the first step.

Step 2. The monoid $M$ in the statement is defined by a system of equations

Let

$$
\mathcal{S}=\{I \varsubsetneqq\{1, \ldots, k\} \mid \text { there exists } a \in A \text { such that } \operatorname{supp}(a)=I\} .
$$

Notice that $\emptyset \in \mathcal{S}$.

For any $I \in \mathcal{S}$, denote by $p_{I}: \mathbb{N}_{0}^{k} \rightarrow \mathbb{N}_{0}^{\{1, \ldots, k\} \backslash I}$ the canonical projection. If $I \in \mathcal{S}$ then, by Proposition 6.1, $A_{I}=p_{I}(A)$ is a full affine submonoid of $\mathbb{N}_{0}^{\{1, \ldots, k\} \backslash I}$.

By step 1 , there is a monoid $M_{I}^{\prime} \subseteq\left(\mathbb{N}_{0}^{*}\right)^{\{1, \ldots, k\} \backslash I}$ defined by a system of equations and such that $\mathbb{N}_{0}^{\{1, \ldots, k\} \backslash I} \cap M_{I}^{\prime}=A_{I}$ and if $x \in M_{I}^{\prime}$ then there exists $a \in A_{I}$ such that $\infty \cdot x=\infty \cdot a$.

Set $M_{I}=\pi_{I}^{-1}\left(M_{I}^{\prime}\right)$. Notice that $M_{I}$ is defined by the same system of equations defining $M_{I}^{\prime}$ but considered over $\mathbb{N}_{0}^{k}$. Notice that $x \in \mathbb{N}_{0}^{k} \cap M_{I}$ if and only if $\pi_{I}(x) \in A_{I}$. In particular, $A=\mathbb{N}_{0}^{k} \cap M_{\emptyset}$ and $A \subseteq M_{I}$ for any $I \in \mathcal{S}$.

Since for any $I \in \mathcal{S}, M_{I}$ is defined by a system of equations so is $\cap_{I \in \mathcal{S}} M_{I}$. We claim that $M=\cap_{I \in \mathcal{S}} M_{I}$. We already know that $A \subseteq \cap_{I \in \mathcal{S}} M_{I}$, so that, by Lemma 6.6(iv), $M \subseteq$ $\cap_{I \in \mathcal{S}} M_{I}$. To prove the other inclusion, let $x \in \cap_{I \in \mathcal{S}} M_{I}$. Let $I_{1}=\inf$-supp $(x)$ and consider the element $x^{*} \in\left(\mathbb{N}_{0}^{*}\right)^{k}$ such that $I_{1}=\inf -\operatorname{supp}\left(x^{*}\right)=\operatorname{supp}\left(x^{*}\right)$. By Lemma 6.6(iii), $x^{*} \in \cap_{I \in \mathcal{S}} M_{I}$. By construction, there exists $a \in A$ such that $x^{*}=\infty \cdot a$, therefore $a \in M$. Since $\operatorname{supp}(a)=I_{1}$, we deduce that $I_{1} \in \mathcal{S}$. Therefore, $\pi_{I_{1}}(x)=\pi_{I_{1}}\left(a_{1}\right)$ for $a_{1} \in A$. This implies that $x=x^{*}+a_{1}$, so that $x \in M$. This finishes the proof of the claim and the proof of the Proposition.

\section{$7 \quad$ Systems of supports}

In order to conclude the proof of Theorem 2.7 we need to show that the monoids that appear as $V^{*}(R)$ for noetherian semilocal rings are defined by a system of equations. To 
this aim we abstract the following class of submonoids of $\left(\mathbb{N}_{0}^{*}\right)^{k}$.

Definition 7.1 Fix $k \in \mathbb{N}$ and an order unit $\left(n_{1}, \ldots, n_{k}\right) \in \mathbb{N}^{k}$. A system of supports $\mathcal{S}\left(n_{1}, \ldots, n_{k}\right)$ consists of a collection $\mathcal{S}$ of subsets of $\{1, \ldots, k\}$ together with a system of monoids $A_{I}, I \in \mathcal{S}$ such that the following conditions hold

(i) $\emptyset \in \mathcal{S}$, and $\left(n_{1}, \ldots, n_{k}\right) \in A_{\emptyset}$.

(ii) For any $I \in \mathcal{S}$ the monoid $A_{I}$ is a submonoid of $\mathbb{N}_{0}^{\{1, \ldots, k\} \backslash I}$. The monoid $A_{\{1, \ldots, k\}}$ is considered as a trivial monoid.

(iii) $\mathcal{S}$ is closed under unions, and if $x \in A_{I}$ for some $I \in \mathcal{S}$ then $I \cup \operatorname{supp}(x) \in \mathcal{S}$.

(iv) Suppose that $I, K \in \mathcal{S}$ are such that $I \subseteq K$ and let $p: \mathbb{N}_{0}^{\{1, \ldots, k\} \backslash I} \rightarrow \mathbb{N}_{0}^{\{1, \ldots, k\} \backslash K}$ be the canonical projection. Then $p\left(A_{I}\right) \subseteq A_{K}$.

If, for any $I \in \mathcal{S}$, the submonoids $A_{I}$ are full affine submonoids of $\mathbb{N}_{0}^{\{1, \ldots, k\} \backslash I}$ then $\mathcal{S}\left(n_{1}, \ldots, n_{k}\right)$ is said to be a full affine system of supports.

In the next Lemma we show that systems of supports are, in some sense, closed under projections.

Lemma 7.2 Let $k>1$, and let $\left(n_{1}, \ldots, n_{k}\right) \in \mathbb{N}^{k}$. Let $\mathcal{S}\left(n_{1}, \ldots, n_{k}\right)=\left(\mathcal{S} ; A_{I}, I \in \mathcal{S}\right)$ be a system of supports. Fix $I \in \mathcal{S} \backslash\{\{1, \ldots, k\}\}$, and let $p:\left(\mathbb{N}_{0}^{*}\right)^{k} \rightarrow\left(\mathbb{N}_{0}^{*}\right)^{\{1, \ldots, k\} \backslash I}$ denote the canonical projection.

If we define $\mathcal{S}_{I}=\{K \backslash I \mid K \in \mathcal{S}$ and $I \subseteq K\}$ and for each $K \backslash I \in \mathcal{S}_{I}$ we take $A_{I, K \backslash I}=A_{K}$, then:

(1) $\mathcal{S}_{I}\left(p\left(n_{1}, \ldots, n_{k}\right)\right)=\left(\mathcal{S}_{I} ; A_{I, K \backslash I}, K \backslash I \in \mathcal{S}_{I}\right)$ is a system of supports of $\left(\mathbb{N}_{0}^{*}\right)^{\{1, \ldots, k\} \backslash I}$.

(2) If $I \neq \emptyset$ then $\left|\mathcal{S}_{I}\right|<|\mathcal{S}|$.

(3) If $\mathcal{S}\left(n_{1}, \ldots, n_{k}\right)$ is a full affine system of supports then so is $\mathcal{S}_{I}\left(p\left(n_{1}, \ldots, n_{k}\right)\right)$.

Proof. It is routine to check that $\mathcal{S}_{I}\left(p\left(n_{1}, \ldots, n_{k}\right)\right)$ satisfies the conditions of a system of supports. Statements (2) and (3) are immediate from the definitions.

We note that the systems of supports is just an alternative way to describe a class submonoids of $\left(\mathbb{N}_{0}^{*}\right)^{k}$,

Proposition 7.3 Fix $k \in \mathbb{N}$ and $\left(n_{1}, \ldots, n_{k}\right) \in \mathbb{N}^{k}$. For any $I \subseteq\{1, \ldots, k\}$, let $p_{I}:\left(\mathbb{N}_{0}^{*}\right)^{k} \rightarrow$ $\left(\mathbb{N}_{0}^{*}\right)^{\{1, \ldots, k\} \backslash I}$ denote the canonical projection. Let $\mathcal{S}\left(n_{1}, \ldots, n_{k}\right)$ be a system of supports. Consider the subset $M(\mathcal{S})$ of $\left(\mathbb{N}_{0}^{*}\right)^{k}$ defined by $x \in M(\mathcal{S})$ if and only if $I=\inf -\operatorname{supp}(x) \in \mathcal{S}$ and $p_{I}(x) \in A_{I}$. Then $M(\mathcal{S})$ is a submonoid of $\left(\mathbb{N}_{0}^{*}\right)^{k}$ such that $\left(n_{1}, \ldots, n_{k}\right) \in \mathbb{N}^{k} \cap M(\mathcal{S})$ and satisfying the properties:

(M1) if $I \subseteq\{1, \ldots, k\}$ is an infinite support of some $x \in M(\mathcal{S})$ then the element $x^{*}$ determined by $\operatorname{supp}\left(x^{*}\right)=\inf -\operatorname{supp}\left(x^{*}\right)=\inf -\operatorname{supp}(x)$ belongs to $M(\mathcal{S})$. 
(M2) If $x \in M(\mathcal{S})$ then $\infty \cdot x \in M(\mathcal{S})$.

Any submonoid $M$ of $\left(\mathbb{N}_{0}^{*}\right)^{k}$ with $M^{k} \cap \mathbb{N}^{k} \neq \emptyset$ and satisfying (M1) and (M2) is of the form $M=M(\mathcal{S})$ for some system of supports $\mathcal{S}$. Moreover, in this situation, for any $I \in \mathcal{S}$, $A_{I}=p_{I}(M) \cap \mathbb{N}_{0}^{\{1, \ldots, k\} \backslash I}$.

Proof. Since $A_{\emptyset} \subseteq M(\mathcal{S}), 0 \in M(\mathcal{S})$ and $\left(n_{1}, \ldots, n_{k}\right) \in M(\mathcal{S})$. To see that $M(\mathcal{S})$ is a monoid it remains to see that it is closed under addition.

Let $x, y \in M(\mathcal{S})$. Set $I=\operatorname{inf-supp}(x), J=\operatorname{inf-supp}(y)$ and $K=I \cup J$. Let $x^{*} \in\left(\mathbb{N}_{0}^{*}\right)^{k}$ be such that $\operatorname{supp}\left(x^{*}\right)=\inf -\operatorname{supp}\left(x^{*}\right)=K$. Notice that $x^{*} \in M(\mathcal{S})$ because $0 \in A_{K}$, and that $x+x^{*}$ and $y+x^{*} \in M(\mathcal{S})$ because of condition (iv) in the definition of system of supports. Then $x+y=\left(x+x^{*}\right)+\left(y+x^{*}\right)$ and, since inf-supp $\left(x+x^{*}\right)=\inf$-supp $\left(y+x^{*}\right)=K$, we deduce that $x+y \in M(\mathcal{S})$.

Note that, since the $A_{I}$ are monoids, $M(\mathcal{S})$ satisfies (M1). Property (M2) follows from condition (iii) in the definition of system of supports.

To prove the converse, let $M$ be a submonoid of $\left(\mathbb{N}_{0}^{*}\right)^{k}$ satisfying (M1) and (M2) and such that $\left(n_{1}, \ldots, n_{k}\right) \in M \cap \mathbb{N}^{k}$. Set

$$
\mathcal{S}=\{I \mid \text { there exists } x \in M \text { such that } \inf -\operatorname{supp}(x)=I\}
$$

as the collection of subsets of $\{1, \ldots, k\}$. Moreover, set $A_{I}=p_{I}(M) \cap \mathbb{N}_{0}^{\{1, \ldots, k\} \backslash I}$ for any $I \in \mathcal{S}$. It is easy to check that the properties of $M$ ensure that $\mathcal{S}\left(n_{1}, \ldots, n_{k}\right)$ is a system of supports such that $M(\mathcal{S})=M$.

Remark 7.4 With the notation as in Proposition 7.3 and Lemma 7.2. assume that $|\mathcal{S}|>2$ and let $\mathcal{T}=\left\{I_{1}, \ldots, I_{\ell}\right\}$ be the set of minimal elements in $\mathcal{S} \backslash\{\emptyset\}$. For each $i \in\{1, \ldots, \ell\}$, let $S_{I_{i}}$ be the system of supports given by Lemma 7.2 and let $M\left(S_{I_{i}}\right)$ be the associated monoid. Then

$$
M(\mathcal{S})=A_{\emptyset} \bigcup\left(\cup_{i=1}^{\ell} M^{\prime}\left(S_{I_{i}}\right)\right)=M_{0} \bigcup\left(\cup_{i=1}^{\ell} M^{\prime}\left(S_{I_{i}}\right)\right)
$$

where $M_{0}=A_{\emptyset}+\left\{\infty \cdot x \mid x \in A_{\emptyset}\right\}$ and

$$
M^{\prime}\left(S_{I_{i}}\right)=\left\{x \in\left(\mathbb{N}_{0}^{*}\right)^{k} \mid p_{I_{i}}(x) \in M\left(S_{I_{i}}\right) \text { and inf-supp }(x) \supseteq I_{i}\right\} .
$$

Now we give a couple of crucial examples of monoids given by a full affine system of monoids.

Example 7.5 Let $k \geq 1$. Let $R$ be a noetherian semilocal ring with an onto ring homomorphism $\varphi: R \rightarrow M_{n_{1}}\left(D_{1}\right) \times \cdots \times M_{n_{k}}\left(D_{k}\right)$ with kernel $J(R)$ and where $D_{1}, \ldots, D_{k}$ are division rings. Let $M=\operatorname{dim}_{\varphi} V^{*}(R) \subseteq\left(\mathbb{N}_{0}^{*}\right)^{k}$. Then $M$ is a submonoid of $\left(\mathbb{N}_{0}^{*}\right)^{k}$ given by a full affine system of supports.

Proof. Notice that $\left(n_{1}, \ldots, n_{k}\right) \in M$. By Theorem 2.3(1), $M$ satisfies condition $(M 1)$ in Proposition 7.3 .

If $P$ is a countably generated projective right $R$-module then $\operatorname{dim}_{\varphi}\left(\left\langle P^{(\omega)}\right\rangle\right)=\infty$. $\operatorname{dim}_{\varphi}(\langle P\rangle)$. Hence $M$ also satisfies condition $(M 2)$ in Proposition 7.3 and we can conclude that $M$ is a submonoid of $\left(\mathbb{N}_{0}^{*}\right)^{k}$ given by a system of supports. 
Let $x \in M$, and let $P$ be a countably generated projective right such that $\operatorname{dim}_{\varphi}(\langle P\rangle)=x$. Let $I=\inf$-supp $(x)$. By Theorem 2.3(1), there exists a countably generated projective right $R$ module $P^{\prime}$ such that

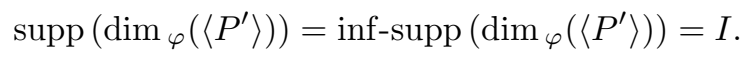

Let $J$ be the trace ideal of $P^{\prime}$. Then $R / J$ is a semilocal ring with Jacobson radical $J+J(R) / J$ and, by Lemma 2.2, $\varphi$ induces an onto ring homomorphism $\bar{\varphi}: R / J \rightarrow \prod_{i \in\{1, \ldots, k\} \backslash I} M_{n_{i}}\left(D_{i}\right)$ with kernel $J(R / J)$. Moreover, by Theorem 2.3(2),

$$
\operatorname{dim}_{\bar{\varphi}}(\langle P / P J\rangle)=p_{I}(x)
$$

where $P / P J$ is a finitely generated projective right $R / J$-module. This shows that $A_{I}=$ $p_{I}(M) \cap \mathbb{N}_{0}^{k} \subseteq \operatorname{dim}_{\bar{\varphi}} V(R / J)$. We claim that $\operatorname{dim}_{\varphi} V(R / J)=A_{I}$. Equivalently, for any finitely generated projective right $R / J$-module $\bar{P}$ there exists a countably generated projective right $R / J$-module $P_{1}$ such that $P_{1} / P_{1} J \cong \bar{P}$. Therefore the claim follows from Proposition 2.1(iii).

By Corollary 1.6. $A_{I}=\operatorname{dim}_{\bar{\varphi}} V(R / J)$ is a full affine submonoid of $\mathbb{N}_{0}^{\{1, \ldots, k\} \backslash I}$. Therefore the monoid $M$ is given by a full affine system of supports as we wanted to show.

Next example is a consequence of Example 7.5 and Theorem 5.3. We prefer to give a proof just in the monoid context.

Example 7.6 Let $D \in M_{n \times k}\left(\mathbb{N}_{0}\right)$ and $E_{1}, E_{2} \in M_{\ell \times k}\left(\mathbb{N}_{0}\right)$. Let $m_{1}, \ldots, m_{n} \in \mathbb{N}$ be such that $m_{i} \geq 2$ for any $i \in\{1, \ldots, n\}$.

Let $M \subseteq\left(\mathbb{N}_{0}^{*}\right)^{k}$ be the set of solution in $\left(\mathbb{N}_{0}^{*}\right)^{k}$ of the system

$$
D\left(\begin{array}{c}
t_{1} \\
\vdots \\
t_{k}
\end{array}\right) \in\left(\begin{array}{c}
m_{1} \mathbb{N}_{0}^{*} \\
\vdots \\
m_{n} \mathbb{N}_{0}^{*}
\end{array}\right) \quad \text { and } \quad E_{1}\left(\begin{array}{c}
t_{1} \\
\vdots \\
t_{k}
\end{array}\right)=E_{2}\left(\begin{array}{c}
t_{1} \\
\vdots \\
t_{k}
\end{array}\right) \text {. }
$$

Assume also that $\left(n_{1}, \ldots, n_{k}\right) \in M \cap \mathbb{N}^{k}$. Then there exists a full affine system of supports $\mathcal{S}\left(n_{1}, \ldots, n_{k}\right)$ such that $M=M(\mathcal{S})$.

Proof. By Lemma 6.6. $M$ satisfies conditions (M1) and (M2) in Proposition 7.3 . Hence, by Proposition 7.3 and following the notation there, $M$ is given by a system of supports in which

$$
\mathcal{S}=\{I \mid \text { there exists } x \in M \text { such that } \inf -\operatorname{supp}(x)=I\}
$$

as a collection of subsets of $\{1, \ldots, k\}$ and, for any $I \in \mathcal{S}, A_{I}=p_{I}(M) \cap \mathbb{N}_{0}^{\{1, \ldots, k\} \backslash I}$. We want to show that, for each $I \in \mathcal{S}$, the monoid $A_{I}$ is full affine in $\mathbb{N}_{0}^{\{1, \ldots, k\} \backslash I}$. To this aim we prove that $A_{I}$ is the set of solution in $\mathbb{N}_{0}^{\{1, \ldots, k\} \backslash I}$ of a certain subsystem of the initial one.

Fix $I \in \mathcal{S}$. Let $D_{I}$ be the matrix with entries in $\mathbb{N}_{0}$ obtained from $D=\left(d_{i j}\right)$ by first deleting the rows $i$ such that there exists $j \in I$ with $d_{i j} \neq 0$, and then deleting in the remaining matrix the $j$-th column for any $j \in I$. Let us denote $K$ the subset of $\{1, \ldots, n\}$ 
indicating which rows of $D$ were deleted and let $p_{K}: \mathbb{N}_{0}^{* n} \rightarrow \mathbb{N}_{0}^{*\{1, \ldots, n\} \backslash K}$ be the canonical projection.

As $I \in \mathcal{S}$, for all $i \in\{1, \ldots, \ell\}$ is either $e_{i, j}^{1}=0=e_{i, j}^{2}$ for all $j \in I$ or there are $j_{1}, j_{2} \in I$ such that $e_{i, j_{1}}^{1} \neq 0$ and $e_{i, j_{2}}^{2} \neq 0$. Let $E_{1}^{I}$ and $E_{2}^{I}$ be the matrices with entries in $\mathbb{N}_{0}$ obtained from $E_{1}=\left(e_{i j}^{1}\right)$ and $E_{2}=\left(e_{i j}^{2}\right)$ by first deleting the rows $i$ such that there exists $j \in I$ satisfying that $e_{i j}^{1}$ is different from zero; after we also delete to each of the remaining matrices the $j$-th column for any $j \in I$.

Then the monoid $A_{I}$ is the set of solutions in $\mathbb{N}_{0}^{\{1, \ldots, k\} \backslash I}$ of the system

$$
D_{I} \cdot p_{I}\left(\begin{array}{c}
t_{1} \\
\vdots \\
t_{k}
\end{array}\right) \in p_{K}\left(\begin{array}{c}
m_{1} \mathbb{N}_{0}^{*} \\
\vdots \\
m_{n} \mathbb{N}_{0}^{*}
\end{array}\right) \quad \text { and } \quad E_{1}^{I} \cdot p_{I}\left(\begin{array}{c}
t_{1} \\
\vdots \\
t_{k}
\end{array}\right)=E_{2}^{I} \cdot p_{I}\left(\begin{array}{c}
t_{1} \\
\vdots \\
t_{k}
\end{array}\right) \text {. }
$$

Theorem 7.7 Let $k \in \mathbb{N}$, and let $\left(n_{1}, \ldots, n_{k}\right) \in \mathbb{N}^{k}$. Let $M$ be a submonoid of $\left(\mathbb{N}_{0}^{*}\right)^{k}$ such that $\left(n_{1}, \ldots, n_{k}\right) \in M$. Then the following statements are equivalent

(i) There exists a full affine system of supports $\mathcal{S}\left(n_{1}, \ldots, n_{k}\right)$ such that $M=M(\mathcal{S})$.

(ii) The submonoid $M$ is defined by a system of equations.

To easy the proof of the theorem we first prove an auxiliary result.

Lemma 7.8 Let $\left(A,\left(n_{1}, \ldots, n_{k}\right)\right) \subseteq\left(\mathbb{N}_{0}^{*}\right)^{k}$ be a monoid defined by a system of equations $\mathcal{E}_{A}$. Let $I$ be a proper nonempty subset of $\{1, \ldots, k\}$ and set $I_{c}=\{1, \ldots, k\} \backslash I$. Let $p:\left(\mathbb{N}_{0}^{*}\right)^{k} \rightarrow\left(\mathbb{N}_{0}^{*}\right)^{I_{c}}$ denote the canonical projection. Assume also $B \subseteq\left(\mathbb{N}_{0}^{*}\right)^{I_{c}}$ is a monoid defined by a system of equations $\mathcal{E}_{B}$ and such that $p\left(n_{1}, \ldots, n_{k}\right) \in B$.

Then the set $A^{\prime} \subseteq\left(\mathbb{N}_{0}^{*}\right)^{k}$ defined by $x=\left(x_{1}, \ldots, x_{k}\right) \in A^{\prime}$ if and only if either

(1) $x \in A$ and $p(x) \in B$

or

(2) $x_{i}=\infty$ for any $i \in I$ and $p(x) \in B$

is a monoid defined by a system of equations. Moreover, $\left(n_{1}, \ldots, n_{k}\right) \in A^{\prime}$.

Proof. Let

$$
D\left(\begin{array}{c}
t_{1} \\
\vdots \\
t_{k}
\end{array}\right) \in\left(\begin{array}{c}
m_{1} \mathbb{N}_{0}^{*} \\
\vdots \\
m_{n} \mathbb{N}_{0}^{*}
\end{array}\right) \quad \text { and } \quad E_{1}\left(\begin{array}{c}
t_{1} \\
\vdots \\
t_{k}
\end{array}\right)=E_{2}\left(\begin{array}{c}
t_{1} \\
\vdots \\
t_{k}
\end{array}\right)
$$

be a system of equations defining $A$. Fix $i \in I$. Let $\mathcal{E}_{A}^{\prime}$ be the system of equations

$$
D\left(\begin{array}{c}
t_{1}+n_{1} t_{i} \\
\vdots \\
t_{k}+n_{k} t_{i}
\end{array}\right) \in\left(\begin{array}{c}
m_{1} \mathbb{N}_{0}^{*} \\
\vdots \\
m_{n} \mathbb{N}_{0}^{*}
\end{array}\right) \quad \text { and } \quad E_{1}\left(\begin{array}{c}
t_{1}+n_{1} t_{i} \\
\vdots \\
t_{k}+n_{k} t_{i}
\end{array}\right)=E_{2}\left(\begin{array}{c}
t_{1}+n_{1} t_{i} \\
\vdots \\
t_{k}+n_{k} t_{i}
\end{array}\right) .
$$


Notice that if $x \in \mathbb{N}_{0}^{k}$ then it is a solution of $\mathcal{E}_{A}^{\prime}$ if and only if $x \in A$; while any $x \in\left(\mathbb{N}_{0}^{*}\right)^{k}$ such that $i \in \inf -\operatorname{supp}(x)$ is a solution of $\mathcal{E}_{A}^{\prime}$.

Let $A_{i} \subseteq\left(\mathbb{N}_{0}^{*}\right)^{k}$ be the solutions of the system $\mathcal{E}_{i}=\mathcal{E}_{A}^{\prime} \cup \mathcal{E}_{B}$, where $\mathcal{E}_{B}$ is the trivial extension of the system defining $B$. Then $x \in \mathbb{N}_{0}^{k}$ belongs to $A_{i}$ if and only if $x \in A$ and $p(x) \in B$. In particular, $\left(n_{1}, \ldots, n_{k}\right) \in A_{i}$. While if $x \in\left(\mathbb{N}_{0}^{*}\right)^{k}$ is such that $i \in \inf$-supp $(x)$ then $x \in A_{i}$ if and only if $p(x) \in B$.

Now the submonoid $A^{\prime}=\cap_{i \in I} A_{i} \subseteq\left(\mathbb{N}_{0}^{*}\right)^{k}$ defined as the solutions of the system $\cup_{i \in I} \mathcal{E}_{i}$, satisfies the properties required in the conclusion of the statement.

Proof of the Theorem. In view of Example 7.6 we only need to prove that (i) implies (ii).

Let $k \in \mathbb{N}$, and let $\mathcal{S}\left(n_{1}, \ldots, n_{k}\right)$ be a full affine system of supports. We proceed by induction on $|\mathcal{S}|$. If $|\mathcal{S}|=2$ then the only sets in $\mathcal{S}$ are $\emptyset$ and $\{1, \ldots, k\}$. So that the only nontrivial full affine semigroup is $A_{\emptyset}$ and, for any $x \in A_{\emptyset} \backslash\{0\}, \operatorname{supp}(x)=\{1, \ldots, k\}$. By [11, Example 2.5], this implies that there exists $y=\left(y_{1}, \ldots, y_{k}\right) \in A_{\emptyset}$ such that $A_{\emptyset}=y \mathbb{N}_{0}$. Therefore $M(\mathcal{S})=A \cup\{\infty \cdot y\}$. By Proposition 6.7, $M(\mathcal{S})$ is defined by a system of equations.

Now assume that $|\mathcal{S}|>2$ and that the statement is true for full affine systems of supports such that the set of supports has smaller cardinality. Let $\mathcal{T} \subseteq \mathcal{S}$ be the set of all minimal elements of $\mathcal{S} \backslash \emptyset$. Note that, since $|\mathcal{S}|>2$, no element in $\mathcal{T}$ is equal to $\{1, \ldots, k\}$. For any $I \in \mathcal{T}$ we construct the full affine system of supports $\mathcal{S}_{I}$ given by Lemma 7.2. As, for each $I \in \mathcal{T},\left|\mathcal{S}_{I}\right|<|\mathcal{S}|$, we know that the monoid $M_{I}\left(\mathcal{S}_{I}\right)$ is given by a system of equations. Moreover, by Proposition 6.7, $M_{0}=A_{\emptyset}+\left\{\infty \cdot x \mid x \in A_{\emptyset}\right\}$ is a submonoid of $\left(\mathbb{N}_{0}^{*}\right)^{k}$ defined by a system of equations.

Let $\mathcal{T}=\left\{I_{1}, \ldots, I_{\ell}\right\}$. We complete $M_{0}$ to a chain $M_{0} \subseteq M_{1} \subseteq \cdots \subseteq M_{\ell}$ of submonoids of $\left(\mathbb{N}_{0}^{*}\right)^{k}$ given by a system of equations, inductively, in the following way: If $i<\ell$ is such that $M_{i}$ is constructed then $M_{i+1}$ is the monoid given by applying Lemma 7.8 to $A=M_{i}$, $I=I_{i+1}$ and $B=M_{I_{i+1}}\left(\mathcal{S}_{I_{i+1}}\right)$. Notice that, following the notation of Lemma 7.8 and by the definition of a system of supports, $p_{I_{i+1}}\left(M_{i}\right) \subseteq M_{I_{i+1}}\left(\mathcal{S}_{I_{i+1}}\right)$, therefore $M_{i+1}=M_{i} \bigcup M_{I_{i+1}}^{\prime}$ where

$$
M_{I_{i+1}}^{\prime}=\left\{x \in\left(\mathbb{N}_{0}^{*}\right)^{k} \mid p_{I_{i+1}}(x) \in M_{I_{i+1}}\left(\mathcal{S}_{I_{i+1}}\right) \text { and inf-supp }(x) \supseteq I_{i+1}\right\} .
$$

Therefore

$$
M_{\ell}=M_{0} \cup M_{I_{1}}^{\prime} \cup \cdots \cup M_{I_{\ell}}^{\prime}=M(\mathcal{S})
$$

by Remark 7.4. This allows us to conclude that $M(\mathcal{S})$ is a monoid given by a system of equations.

Now Theorem 2.7 follows by just patching together our previous results.

Proof Theorem 2.7, (1) $\Rightarrow(2)$ follows from Theorem [5.3 It is clear that (2) $\Rightarrow(3)$.

Finally, assume (3). By Example 7.5, $M$ is given by a full affine system of supports. By Theorem $7.7 M$ is defined by a system of equations, and (1) follows. 


\section{Some consequences and examples}

In order to be able to construct further examples, first we single out the monoids corresponding to semilocal rings such that any projective right $R$-module is a direct sum of finitely generated modules. They are precisely the ones arising in Proposition 6.7.

Corollary 8.1 Let $k \in \mathbb{N}$. Let $M$ be a submonoid of $\left(\mathbb{N}_{0}^{*}\right)^{k}$ containing $\left(n_{1}, \ldots, n_{k}\right) \in \mathbb{N}^{k}$. Let $A=M \cap \mathbb{N}_{0}^{k}$. Then the following statements are equivalent:

(i) $A$ is a full affine submonoid of $\mathbb{N}_{0}^{k}$ and $M=A+\{\infty \cdot a \mid a \in A\}$.

(ii) There exists a (noetherian) semilocal ring $R$ such that all projective right $R$ modules are direct sum of finitely generated modules, and an onto morphism $\varphi: R \rightarrow$ $M_{n_{1}}\left(D_{1}\right) \times \cdots \times M_{n_{k}}\left(D_{k}\right)$ with $\operatorname{Ker} \varphi=J(R)$, where $D_{1}, \ldots, D_{k}$ are suitable division rings, satisfying that $\operatorname{dim}_{\varphi} V^{*}(R)=M$.

Proof. Assume $(i)$. By Proposition 6.7 $M$ is given by a system of equations. By Theorem 2.7 (ii) holds.

Assume (ii). So that $R$ is a, not necessarily noetherian, semilocal ring such that all projective right modules are direct sum of finitely generated ones. By Corollary 1.6, $A$ is a full submonoid of $\mathbb{N}_{0}^{k}$. It is clear that $A+\{\infty \cdot a \mid a \in A\} \subseteq M$. Let $P_{1}, \ldots, P_{s}$ be a set of representatives of the indecomposable (hence finitely generated) projective right modules. For $i=1, \ldots, s$, let $a_{i}=\operatorname{dim}_{\varphi}\left(\left\langle P_{i}\right\rangle\right)$. As any projective module is a direct sum of finitely generated projective modules, any $x \in M$ satisfies that $x=\alpha_{1} a_{1}+\cdots+\alpha_{s} a_{s}$ for some $\alpha_{i} \in \mathbb{N}_{0}^{*}$, hence $x \in A+\{\infty \cdot a \mid a \in A\}$. This shows that (i) holds.

In Examples 8.5 we shall see that the property that all projective modules are direct sum of finitely generated ones is not left right symmetric. By Theorem 2.3 it is left-right symmetric in the noetherian semilocal case.

We stress the fact that, in view of Theorem 2.7, noetherian semilocal rings can have a rich supply of infinitely generated projective modules that are not direct sum of finitely generated ones.

It is quite an interesting question to determine the structure of $V^{*}(R)$ for a general semilocal ring. But right now it seems to be too challenging even for semilocal rings $R$ such that $R / J(R) \cong D_{1} \times D_{2}$ for $D_{1}, D_{2}$ division rings. Now we provide some examples of such rings to illustrate Theorem 2.7 and the difficulties that appear in the general case. We first observe that with such rings, since $k=2$ and $(1,1)$ is the order unit of $V(R)$, to have some room for interesting behavior all finitely generated projective modules must be free.

Lemma 8.2 Let $R$ be a semilocal ring such that $R / J(R) \cong D_{1} \times D_{2}$ for suitable division rings $D_{1}$ and $D_{2}$. Fix $\varphi: R \rightarrow D_{1} \times D_{2}$ an onto ring homomorphism such that $\operatorname{Ker} \varphi=J(R)$. If $R$ has nonfree finitely generated projective right (or left) modules then there exists $n \in \mathbb{N}$ such that $\operatorname{dim}_{\varphi} V(R)$ is the submonoid of $\mathbb{N}_{0}^{2}$ generated by $(1,1),(n, 0)$ and $(0, n)$. In this case,

$$
\operatorname{dim}_{\varphi} V^{*}(R)=(1,1) \mathbb{N}_{0}^{*}+(n, 0) \mathbb{N}_{0}^{*}+(0, n) \mathbb{N}_{0}^{*}=\left\{(x, y) \in \mathbb{N}_{0}^{*} \mid x+(n-1) y \in n \mathbb{N}_{0}^{*}\right\} .
$$


Therefore, all projective modules are direct sum of finitely generated projective modules.

Proof. Note that $\operatorname{dim}_{\varphi}(\langle R\rangle)=(1,1)$. So that $(1,1) \in A=\operatorname{dim}_{\varphi} V(R)$.

Let $P$ be a nonfree finitely generated projective right $R$-module, and let $\operatorname{dim}_{\varphi}(\langle P\rangle)=$ $(x, y)$. As $P$ is not free, either $x>y$ or $x<y$. Assume $x>y$, the other case is done in a symmetric way. Then

$$
(x, y)=(x-y, 0)+y(1,1) \in A
$$

since, by Corollary 1.6. $A$ is a full affine submonoid of $\mathbb{N}_{0}^{2}$ we deduce that $(x-y, 0) \in A$ and also that $(0, x-y)=(x-y)(1,1)-(x-y, 0) \in A$. Choose $n \in \mathbb{N}$ minimal with respect to the property $(n, 0) \in A$, and note that then also $(0, n) \in A$. We claim that

$$
A=(1,1) \mathbb{N}_{0}+(n, 0) \mathbb{N}_{0}+(0, n) \mathbb{N}_{0} .
$$

We only need to prove that if $(x, y) \in A$ then it can be written as a linear combination, with coefficients in $\mathbb{N}_{0}$ of $(1,1),(n, 0)$ and $(0, n)$. In view of the previous argument, it suffices to show that if $(x, 0) \in A$ then $(x, 0) \in(n, 0) \mathbb{N}_{0}$. By the division algorithm $(x, 0)=$ $(n, 0) q+(r, 0)$ with $q \in \mathbb{N}_{0}$ and $0 \leq r<n$. As $A$ is a full affine submonoid of $\mathbb{N}_{0}^{2}$ we deduce that $(r, 0) \in A$. By the choosing of $n$ we can deduce that $r=0$, as desired.

Let $P_{1}$ be a finitely generated right $R$-module such that $\operatorname{dim}_{\varphi}\left(\left\langle P_{1}\right\rangle\right)=(n, 0)$, and let $P_{2}$ be a finitely generated right $R$-module such that $\operatorname{dim}_{\varphi}\left(\left\langle P_{2}\right\rangle\right)=(0, n)$.

Let $Q$ be a countably generated projective right $R$-module that is not finitely generated. Let $\operatorname{dim}_{\varphi}(\langle Q\rangle)=(x, y) \in \mathbb{N}_{0}^{*}$. We want to show that

$$
(x, y) \in(1,1) \mathbb{N}_{0}^{*}+(n, 0) \mathbb{N}_{0}^{*}+(0, n) \mathbb{N}_{0}^{*}
$$

If $x=y$ then $(x, y)=x(1,1)$ and, by Theorem 1.4(ii), $Q$ is free. If $x>y$ then $y \in \mathbb{N}_{0}$ and $(x, y)=(x-y, 0)+y(1,1)$, combining Theorem 1.4(ii) with Lemma 1.3 we deduce that $Q=y R \oplus Q^{\prime}$ with $Q^{\prime}$ such that $\operatorname{dim}_{\varphi}\left(\left\langle Q^{\prime}\right\rangle\right)=(z, 0)$ where $z=x-y$. If $z<\infty$ then, by Theorem 1.4(ii), $n Q^{\prime} \cong z P_{1}$ hence $Q^{\prime}$, and $Q$, are finitely generated. So that, $z=\infty$ and then, by Theorem 1.4(ii), $Q^{\prime} \cong P_{1}^{(\omega)}$. Hence $(x, y)=\infty \cdot(n, 0)+y(1,1)$. The case $x<y$ is done in a symmetric way.

It is not difficult to check that the elements of $\operatorname{dim}_{\varphi} V^{*}(R)$ are the solutions in $\mathbb{N}_{0}^{*}$ of $x+(n-1) y \in n \mathbb{N}_{0}^{*}$.

Now we list all the possibilities for the monoid $V^{*}(R)$ viewed as a submonoid of $V^{*}(R / J(R))$ when $R$ is a noetherian ring such that $R / J(R) \cong D_{1} \times D_{2}$, for $D_{1}$ and $D_{2}$ division rings, and all finitely generated projective modules are free.

We recall that a module is superdecomposable if it has no indecomposable direct summand. By Theorem 2.7 and Lemma 1.3. in our context superdecomposable modules are relatively frequent as they correspond to the elements $x$ in the monoid $M \subseteq\left(\mathbb{N}_{0}^{*}\right)^{k}$ such that for any $y \in M \cap \mathbb{N}_{0}^{k} \operatorname{supp}(y) \nsubseteq \operatorname{supp}(x)$.

Example 8.3 Let $R$ be a semilocal noetherian ring such that there exists $\varphi: R \rightarrow D_{1} \times D_{2}$, an onto ring morphism with $\operatorname{Ker} \varphi=J(R)$, where $D_{1}$ and $D_{2}$ are division rings. Assume 
that all finitely generated projective right $R$-modules are free. Hence $\operatorname{dim}_{\varphi} V(R)=(1,1) \mathbb{N}_{0}$, and its order unit is $(1,1)$. Then there are the following possibilities for $\operatorname{dim}_{\varphi} V^{*}(R)$ :

(0) All projective modules are free, so that $M_{0}=\operatorname{dim}_{\varphi} V^{*}(R)=(1,1) \mathbb{N}_{0}^{*}$. Note that $M_{0}$ is the set of solutions $(x, y) \in \mathbb{N}_{0}^{*}$ of the equation $x=y$.

(1) $M_{1}=\operatorname{dim}_{\varphi} V^{*}(R)=(1,1) \mathbb{N}_{0}^{*}+(0, \infty) \mathbb{N}_{0}^{*}$. So that, $M_{1}$ is the set of solutions $(x, y) \in$ $\mathbb{N}^{*}$ of the equation $x+y=2 y$.

Note that for such an $R$ there exists a countably generated superdecomposable projective right $R$-module $P$ such that $\operatorname{dim}_{\varphi}(\langle P\rangle)=(0, \infty)$. Then any countably generated projective right $R$ module $Q$ satisfies that there exist $n \in \mathbb{N}_{0}^{*}$ and $m \in\{0,1\}$ such that $Q=R^{(n)} \oplus P^{(m)}$.

(1') $M_{1}^{\prime}=\operatorname{dim}_{\varphi} V^{*}(R)=(1,1) \mathbb{N}_{0}^{*}+(\infty, 0) \mathbb{N}_{0}^{*}$. So that, $M_{1}^{\prime}$ is the set of solutions $(x, y) \in$ $\mathbb{N}^{*}$ of the equation $x+y=2 x$.

(2) $M_{2}=\operatorname{dim}_{\varphi} V^{*}(R)=(1,1) \mathbb{N}_{0}^{*}+(\infty, 0) \mathbb{N}_{0}^{*}+(0, \infty) \mathbb{N}_{0}^{*}$. So that, $M_{2}$ is the set of solutions $(x, y) \in \mathbb{N}_{0}^{*}$ of the equation $2 x+y=x+2 y$.

Note that for such an $R$ there exist two countably generated superdecomposable projective right $R$-modules $P_{1}$ and $P_{2}$ such that $\operatorname{dim}_{\varphi}\left(\left\langle P_{1}\right\rangle\right)=(0, \infty)$ and $\operatorname{dim}_{\varphi}\left(\left\langle P_{2}\right\rangle\right)=$ $(\infty, 0)$. Any countably generated projective right $R$ module $Q$ satisfies that there exist $n \in \mathbb{N}_{0}$ and $m_{1}, m_{2} \in\{0,1\}$ such that $Q=R^{(n)} \oplus P_{1}^{\left(m_{1}\right)} \oplus P_{2}^{\left(m_{2}\right)}$.

Proof. In view of Theorem 2.7. Theorem 7.7 and Lemma 8.2 we must describe all the possibilities for full affine systems of supports of $\{1,2\}$ such that $A_{\emptyset}=(1,1) \mathbb{N}_{0}$. Since the set of supports of a system of supports at least contains $\emptyset$ and $\{1,2\}$ there are just four possibilities.

Since the image of the projections of $A_{\emptyset}$ either on the first or onto the second component is $\mathbb{N}_{0}$, all the monoids $A_{I}$ in the definition of system of supports are determined by $A_{\emptyset}$.

Case (0) is the one in which $M_{0}=A_{\emptyset}+\infty \cdot A_{\emptyset}$. According to Corollary 8.1, in this case all projective modules are direct sum of finitely generated (indecomposable) modules.

In cases (1) and $\left(1^{\prime}\right)$ there are 3 different infinite supports for the elements in the monoid, and in case (2) there are 4 .

The monoid $V^{*}(R)$ for non noetherian rings $R$ with exactly two maximal right ideals can have a more complicated structure. One of the reasons is that it may happen that $V(R) \varsubsetneqq W(R)$, cf. [1.6 In the next Theorem we recall the almost only existing family of such examples until now.

Note that, by Proposition 1.8, for a semilocal ring with a fixed onto map $\varphi: R \rightarrow$ $M_{n_{1}}\left(D_{1}\right) \times \cdots \times M_{n_{k}}\left(D_{k}\right)$ to a semisimple artinian ring with kernel $J(R)$

$W(R)=\left\{\langle P\rangle \in V^{*}(R) \mid\langle P / P J(R)\rangle \in V(R / J(R))\right\} \quad$ and $\quad \operatorname{dim}_{\varphi} W(R)=\left(\operatorname{dim}_{\varphi} V^{*}(R)\right) \cap \mathbb{N}_{0}^{k}$.

We point out the fact that we do not even know whether the monoid $W(R)$ must be finitely generated. 
Theorem 8.4 Let $F$ be any field. Then there exists a semilocal $F$-algebra $R$ with an onto ring morphism $\varphi: R \rightarrow F \times F$ with $\operatorname{Ker} \varphi=J(R)$ and such that all finitely generated projective modules are free but

$$
\begin{aligned}
\operatorname{dim}_{\varphi} W\left(R_{R}\right) & =\left\{(x, y) \in \mathbb{N}_{0} \mid x \geq y\right\}=(1,1) \mathbb{N}_{0}+(1,0) \mathbb{N}_{0} \\
\operatorname{dim}_{\varphi} V^{*}\left(R_{R}\right) & =\left(\operatorname{dim}_{\varphi} W\left(R_{R}\right)\right) \mathbb{N}_{0}^{*}
\end{aligned}
$$

and

$$
\begin{aligned}
\operatorname{dim}_{\varphi} W\left({ }_{R} R\right) & =\left\{(x, y) \in \mathbb{N}_{0} \mid y \geq x\right\}=(1,1) \mathbb{N}_{0}+(0,1) \mathbb{N}_{0} \\
\operatorname{dim}_{\varphi} V^{*}\left({ }_{R} R\right) & =\left(\operatorname{dim}_{\varphi} W\left({ }_{R} R\right)\right) \mathbb{N}_{0}^{*}
\end{aligned}
$$

Proof. One possibility for such an example is the one constructed by Gerasimov and Sakhaev in [16. The monoids $V^{*}\left(R_{R}\right)$ and $V^{*}\left({ }_{R} R\right)$ were computed in 8 .

Another possibility for such an example is the endomorphism ring of a nonstandard uniserial module. Such modules were constructed by Puninski in [25], and it was observed in 12 that the endomorphism ring of such modules have such pathological projective modules. The monoids were computed in [22, Theorem 4.7].

Next family of examples shows that the combination of Theorem 8.4 with the pullback constructions gives a good tool to construct further examples.

The duality between finitely generated projective right modules and finitely generated projective left modules does not extend to an isomorphism between $W\left(R_{R}\right)$ and $W\left({ }_{R} R\right)$. But, as it follows from Proposition 1.8, in the semilocal case, one determines the other.

In the next Example we show that, in general, for semilocal rings the monoids $V^{*}\left({ }_{R} R\right)$ and $V^{*}\left(R_{R}\right)$ not only are not isomorphic but also that one monoid does not determine the other. We do that by giving an example of a semilocal ring $R$ such that all projective left modules are free but $V^{*}\left(R_{R}\right)$ is isomorphic to the monoid in Example 8.3(1'); in particular, not all right projective modules are direct sum of finitely generated ones.

Example 8.5 Let $F$ be any field. In all the statements $R$ denotes a semilocal $F$-algebra, and $\varphi: R \rightarrow F \times F$ denotes an onto ring homomorphism such that $\operatorname{Ker} \varphi=J(R)$. Fix $n \in \mathbb{N}$. Then:

(i) There exist $R$ and $\varphi$ such that

$$
\begin{aligned}
& N_{1}=\operatorname{dim}_{\varphi} V^{*}\left(R_{R}\right)=(1,1) \mathbb{N}_{0}^{*}+(n, 0) \mathbb{N}_{0}^{*}=\left\{(x, y) \in\left(\mathbb{N}_{0}^{*}\right)^{2} \mid x \geq y \text { and } x+(n-1) y \in n \mathbb{N}_{0}^{*}\right\} \\
& N_{2}=\operatorname{dim}_{\varphi} V^{*}\left({ }_{R} R\right)=(1,1) \mathbb{N}_{0}^{*}+(0, n) \mathbb{N}_{0}^{*}=\left\{(x, y) \in\left(\mathbb{N}_{0}^{*}\right)^{2} \mid x \leq y \text { and } x+(n-1) y \in n \mathbb{N}_{0}^{*}\right\}
\end{aligned}
$$

(ii) There exist $R$ and $\varphi$ such that

$$
\begin{aligned}
& \operatorname{dim}_{\varphi} V^{*}\left(R_{R}\right)=N_{1}+(0, \infty) \mathbb{N}_{0}^{*}=\left\{(x, y) \in\left(\mathbb{N}_{0}^{*}\right)^{2} \mid 2 x+y \geq 2 y+x \text { and } x+(n-1) y \in n \mathbb{N}_{0}^{*}\right\} \\
& \operatorname{dim}_{\varphi} V^{*}\left({ }_{R} R\right)=N_{2}+(\infty, 0) \mathbb{N}_{0}^{*}=\left\{(x, y) \in\left(\mathbb{N}_{0}^{*}\right)^{2} \mid 2 x+y \leq 2 y+x \text { and } x+(n-1) y \in n \mathbb{N}_{0}^{*}\right\}
\end{aligned}
$$

(iii) There exists $R$ and $\varphi$ such that $\operatorname{dim}_{\varphi} V^{*}\left(R_{R}\right)=(1,1) \mathbb{N}_{0}^{*}+(\infty, 0) \mathbb{N}_{0}^{*}$ and $\operatorname{dim}_{\varphi} V^{*}\left({ }_{R} R\right)=$ $(1,1) \mathbb{N}_{0}^{*}$. Therefore, all projective left $R$-modules are direct sum of finitely generated modules but this is not true for projective right $R$-modules. In particular, $V^{*}\left(R_{R}\right)$ and $V^{*}\left({ }_{R} R\right)$ are not isomorphic. 
Proof. To construct $(i)$, let $R_{1}$ be an $F$ algebra with an onto ring homomorphism $j_{1}: R_{1} \rightarrow D_{1} \times D_{2}$ where $\operatorname{Ker} j_{1}=J(R)$ and $D_{1}, D_{2}$ are division rings. Moreover, assume that $\operatorname{dim}_{j_{1}} V^{*}(R)=(1,1) \mathbb{N}_{0}^{*}+(n, 0) \mathbb{N}_{0}^{*}+(0, n) \mathbb{N}_{0}^{*}=M$, cf. Lemma 8.2 The existence of $R_{1}$ and $j_{1}$ is a consequence either of Theorem 2.7 or of [10, Theorem 6.1].

Let $R_{2}$ and $j_{2}: R_{2} \rightarrow F \times F$ be ring and the onto map, respectively, given by Theorem 8.4 That is,

$$
M_{1}=\operatorname{dim}_{j_{2}} V^{*}\left(R_{2 R_{2}}\right)=\left\{(x, y) \in \mathbb{N}_{0}^{*} \mid x \geq y\right\}=(1,1) \mathbb{N}_{0}^{*}+(1,0) \mathbb{N}_{0}^{*}
$$

and

$$
M_{2}=\operatorname{dim}_{j_{2}} V^{*}\left(R_{2} R_{2}\right)=\left\{(x, y) \in \mathbb{N}_{0}^{*} \mid y \geq x\right\}=(1,1) \mathbb{N}_{0}^{*}+(0,1) \mathbb{N}_{0}^{*} .
$$

As $F \times F \subseteq D_{1} \times D_{1}$, we extend $j_{2}: R_{2} \rightarrow D_{1} \times D_{2}$ and consider the pullback

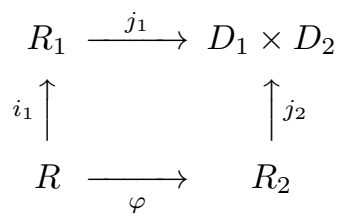

By Corollary 4.9 and Corollary 4.10 $R$ is a semilocal $F$-algebra, $\varphi$ is an onto ring homomorphism with kernel $J(R)$ and

$$
\operatorname{dim}_{\varphi} V^{*}\left(R_{R}\right)=M \cap M_{1} \quad \operatorname{dim}_{\varphi} V^{*}\left({ }_{R} R\right)=M \cap M_{2}
$$

which are the monoids $N_{1}$ and $N_{2}$ respectively.

To construct example (ii) set $R_{1}$ and $j_{1}: R_{1} \rightarrow F \times F$ to be the ring and the onto ring homomorphism, respectively, constructed in $(i)$. Let $j_{2}: F \times F \rightarrow M_{3}(F) \times M_{3}(F)$ be the map defined by

$$
j_{2}(x, y)=\left(\left(\begin{array}{lll}
x & 0 & 0 \\
0 & x & 0 \\
0 & 0 & y
\end{array}\right),\left(\begin{array}{lll}
y & 0 & 0 \\
0 & x & 0 \\
0 & 0 & y
\end{array}\right)\right)
$$

Note that $j_{2}$ induces the monoid homomorphism $f:\left(\mathbb{N}_{0}^{*}\right)^{2} \rightarrow\left(\mathbb{N}_{0}^{*}\right)^{2}$ defined by $f(x, y)=$ $(2 x+y, 2 y+x)$.

Let $R$ be the ring defined by the pullback

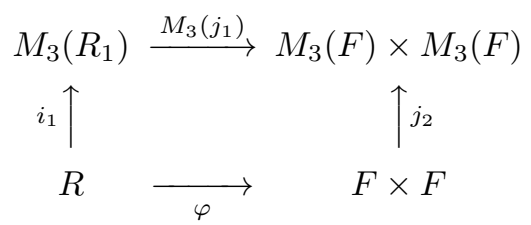

By Corollary 4.9, $R$ is a semilocal $F$-algebra, $\varphi$ is an onto ring homomorphism with kernel $J(R)$, and $\operatorname{dim}_{\varphi} V^{*}\left(R_{R}\right)$ and $\operatorname{dim}_{\varphi} V^{*}\left({ }_{R} R\right)$ are as claimed in $(i i)$.

To construct example (iii), let $R_{1}$ and $j_{1}: R_{1} \rightarrow F \times F$ be ring and the onto map, respectively, given by Theorem 8.4. Let $R_{2}$ be a semilocal ring with an onto ring homomorphism $j_{2}: R_{2} \rightarrow F \times F$, with Ker $j_{2}=J\left(R_{2}\right)$, satisfying that $\operatorname{dim}_{j_{2}} V^{*}\left(\left(R_{2}\right)_{R_{2}}\right)=$ $\operatorname{dim}_{j_{2}} V^{*}\left(R_{2} R_{2}\right)=(1,1) \mathbb{N}_{0}^{*}+(\infty, 0) \mathbb{N}_{0}^{*}$. Note that such example exists by Example 8.3 $(1)$. 
Let $R$ be the ring defined by the pullback

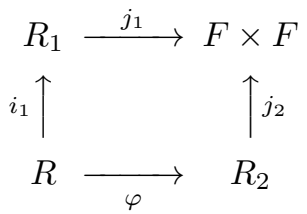

By Corollary 4.10, $R$ is a semilocal $F$-algebra, $j_{2} \varphi$ is an onto ring homomorphism with kernel $J(R)$ and

$$
\begin{gathered}
\operatorname{dim}_{j_{2} \varphi} V^{*}\left(R_{R}\right)=\left((1,1) \mathbb{N}_{0}^{*}+(1,0) \mathbb{N}_{0}^{*}\right) \cap\left((1,1) \mathbb{N}_{0}^{*}+(\infty, 0) \mathbb{N}_{0}^{*}\right)=(1,1) \mathbb{N}_{0}^{*}+(\infty, 0) \mathbb{N}_{0}^{*} \\
\operatorname{dim}_{j_{2} \varphi} V^{*}\left({ }_{R} R\right)=\left((1,1) \mathbb{N}_{0}^{*}+(0,1) \mathbb{N}_{0}^{*}\right) \cap\left((1,1) \mathbb{N}_{0}^{*}+(\infty, 0) \mathbb{N}_{0}^{*}\right)=(1,1) \mathbb{N}_{0}^{*}
\end{gathered}
$$

Therefore all projective left $R$-modules are free. On the other hand if $P$ is a countably generated projective right $R$-module such that $\operatorname{dim}_{j_{2} \varphi}(\langle P\rangle)=(\infty, 0)$ then $P$ is superdeccomposable, hence, it is not a direct sum of finitely generated projective right $R$-modules. In order to see that $V^{*}\left(R_{R}\right)$ and $V^{*}\left({ }_{R} R\right)$ are not isomorphic, it is enough to count idempotents in these monoids.

Remark 8.6 Example 8.5(ii) answers a problem mentioned in [8, page 3261], and Example 8.5(iii) answers a problem in [15, page 310].

It follows from Proposition 1.8 that Corollary 2.4 fails for general semilocal rings, as the order induced on $W(R)$, viewed as a submonoid of $V^{*}(R / J(R))$, is not the algebraic order. Next example shows that this can fail also for the subsemigroup $W(R) \backslash V(R)$.

Example 8.7 For any field $F$, there exists a semilocal $F$-algebra $R$ and an onto morphism $\varphi: R \rightarrow F \times F \times F$, with $\operatorname{Ker} \varphi=J(R)$, satisfying that

$$
\begin{aligned}
& M_{1}=\operatorname{dim}_{\varphi} V^{*}\left(R_{R}\right)=\left\{(x, y, z) \in\left(\mathbb{N}_{0}^{*}\right)^{3} \mid x \geq y \text { and } y \geq z\right\} \\
& M_{2}=\operatorname{dim}_{\varphi} V^{*}\left({ }_{R} R\right)=\left\{(x, y, z) \in\left(\mathbb{N}_{0}^{*}\right)^{3} \mid y \geq x \text { and } z \geq y\right\}
\end{aligned}
$$

In particular, $a=(1,1,0)$ and $b=(1,0,0) \in M_{1}$, clearly $b<a$, but there is no element $b^{\prime} \in M_{1}$ such that $b+b^{\prime}=a$.

Proof. Let $T$ be the $F$-algebra given by Theorem 8.4. Set $R_{1}=T \times T$. Let $j_{1}: R_{1} \rightarrow F^{4}$ be an onto ring homomorphism with kernel $J\left(R_{1}\right)=J(T) \times J(T)$. Let $j_{2}: F \times F \times F \rightarrow F^{4}$ be the morphism defined by $j_{2}(x, y, z)=(x, y, y, z)$.

Note that $j_{2}$ induces the morphism of monoids $f:\left(\mathbb{N}_{0}^{*}\right)^{3} \rightarrow\left(\mathbb{N}_{0}^{*}\right)^{4}$ also defined by $f(x, y, z)=(x, y, y, z)$.

Let $R$ be the ring defined by the pullback diagram

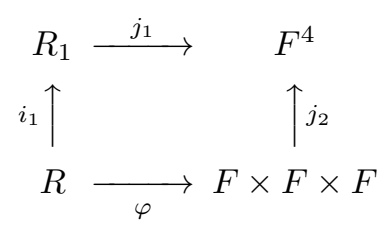


By Corollary 4.9, $R$ is a semilocal $F$-algebra and $\varphi$ is an onto ring homomorphism with kernel $J(R)$. We have chosen $R_{1}$ such that $\operatorname{dim}_{j_{1}} V^{*}\left(\left(R_{1}\right)_{R_{1}}\right)=\left\{(x, y, z, t) \in\left(\mathbb{N}_{0}^{*}\right)^{4} \mid x \geq\right.$ $y$ and $z \geq t\}$. By Corollary 4.9 $(x, y, z) \in \operatorname{dim}_{\varphi} V^{*}\left(R_{R}\right)$ if and only if $x \geq y \geq z$ as desired. Similarly, as $\operatorname{dim}_{j_{1}} V^{*}\left(R_{1} R_{1}\right)=\left\{(x, y, z, t) \in\left(\mathbb{N}_{0}^{*}\right)^{4} \mid x \leq y\right.$ and $\left.z \leq t\right\}, \operatorname{dim}_{\varphi} V^{*}\left({ }_{R} R\right)=$ $\left\{(x, y, z) \in\left(\mathbb{N}_{0}^{*}\right)^{3} \mid x \leq y\right.$ and $\left.y \leq z\right\}$. The rest of the statement is clear.

\section{References}

[1] H. Bass, Big Projective modules are free, Illinois J. of Math. 7(1963), 24-31.

[2] W. Bruns, J. Gubeladze, "Polytopes, rings and $K$-theory", To be published by Springer. Available at: http://www.mathematik.uni-osnabrueck.de/staff/phpages/brunsw.rdf.shtml

[3] W. Bruns, J. Herzog, "Cohen-Macaulay rings", Cambridge studies in Advanced Mathematics 39, Cambridge University Press, Cambridge, 1996.

[4] M.C.R. Butler, J. M. Campbell, L. G. Kovács, On infinite rank integral representations of groups and orders of finite lattice type,Arch. Math. (Basel) 83 (2004), no. 4, 297-308.

[5] R. Camps and W. Dicks, On semilocal rings, Israel J. Math. 81 (1993), 203-211.

[6] L. Chiunard, Krull semigroups and divisor class groups, Canadian J. Math. 19 (1981), 14591468.

[7] K. T. Coward, G. A. Elliott, C. Ivanescu, The Cuntz semigroup as an invariant for $C^{*}$-algebras, J. Reine Angew. Math. 623(2008), 161-193.

[8] N. Dubrovin, P. Př́hoda, G. Puninski, Projective modules over the Gerasimov-Sakhaev counterexample J. Algebra 319 (2008), no. 8, 3259-3279.

[9] A. Facchini, "Module Theory. Endomorphism rings and direct sum decompositions in some classes of modules", Progress in Math. 167, Birkhäuser Verlag, Basel, 1998.

[10] A. Facchini, D. Herbera, $K_{0}$ of a semilocal ring, J. Algebra 225 (2000), 47-69.

[11] A. Facchini and D. Herbera, Projective modules over semilocal rings, in "Algebra and its Applications", D. V. Huynh, S. K. Jain, S. R. López-Permouth eds., Contemporary Math. 259, Amer. Math. Soc., Providence, 2000, pp. 181-198.

[12] A. Facchini, D. Herbera and I. Sakhaev, Finitely Generated Flat Modules and a Characterization of Semiperfect Rings, Comm. in Alg. 31 (2003), 4195-4214.

[13] A. Facchini, D. Herbera and I. Sakhaev, Flat modules and lifting of finitely generated projective modules, Pacific J. Math. 220 (2005), 49-67.

[14] A. Facchini and D. Herbera, Local Morphisms and Modules with a Semilocal Endomorphism Ring, Algebras and Rep. Theory 9 (2006), pp. 403-422.

[15] K. Fuller, W. Shutters, Projective modules over non-commutative semilocal rings, Tohoku Math. Journ. 27 (1975), 303-311.

[16] V. N. Gerasimov and I. I. Sakhaev, A counterexample to two hypotheses on projective and flat modules (Russian), Sib. Mat. Zh. 25 (6) (1984), 31-35. English translation: Sib. Math. J. 24 (1984), 855-859.

[17] D. Herbera and A. Shamsuddin, Modules with semi-local endomorphism ring, Proc. Amer. Math. Soc. 123 (1995), 3593-3600.

[18] Y. Hinohara, Projective modules over semilocal rings, Tôhoku Math. J. 14 (1962), 205-211.

[19] M. Hochster, Rings of invariants of tori, Cohen-Macaulay rings generated by monomials, and polytopes, Ann. of Math. 96 (1972), 318-337.

[20] D. Lazard, Liberté des Gros Modules Projectives J. Algebra 31 (1974), 437-451. 
[21] John Milnor, "Introduction to Algebraic K-Theory", Anals of Mathematics Studies 72, Princeton University Press, 1971.

[22] P. Příhoda, Projective modules are determined by their radical factors, J. Pure Applied Algebra 210 (2007), $827-835$.

[23] P. Příhoda, Fair-sized projective modules, http://artax.karlin.mff.cuni.cz/ ppri7485/temp/fsp.pdf

[24] J. M. Whitehead, Projective modules and their trace ideals, Comm. Algebra 8(19) (1980), 1873-1901.

[25] G. Puninski, Some model theory over a nearly simple uniserial domain and decompositions of serial modules, J. Pure Appl. Algebra 2001, 163, 319-337.

[26] W. Rump, Large lattices over orders, Proc. London Math. Soc. (3) 91 (2005), no. 1, 105-128.

[27] I. I. Sakhaev, Lifting the finite generation of a projective module modulo its radical, Mat. Zametki 49 (1991), 97-108. English translation: Math. Notes 49 (1991), 291-301.

[28] R. Wiegand, Direct sum Decompositions over Local Rings, J. Algebra 240 (2001), 83-97.

[29] R. Wiegand and S. Wiegand, Semigroups of Modules: A survey, to appear in the Proceedings of the International Conference on Rings and Things.

[30] A. V. Yakovlev, On direct decompositions of p-adic groups, (Russian) Algebra i Analiz 12 (2000), no. 6, 217-223; English translation: St. Petersburg Math. J. 12 (2001), no. 6, 10431047.

[31] A. V. Yakovlev, On direct decompositions of S-local groups, (Russian) Algebra i Analiz 13 (2001), no. 4, 229-253; English translation: St. Petersburg Math. J. 13 (2002), no. 4, 685-702. 